\title{
Development of a HIL railway roller rig model for the traction and braking testing activities under degraded adhesion conditions
}

\author{
B. Allotta, R. Conti*, E. Meli, L. Pugi, A. Ridolfi \\ Department of Industrial Engineering, University of Florence, Via S. Marta n. 3, 50139 Florence, Italy
}

\section{A R T I C L E I N F O}

\section{Article history:}

Received 11 March 2013

Accepted 4 June 2013

Available online 27 June 2013

Keywords:

Multibody model of railway vehicles

HIL

Fullscale roller rig

Degraded adhesion

WSP (Wheel Slide Protection)

Antiskid

\begin{abstract}
A B S T R A C T
The longitudinal train dynamics is controlled by traction and braking on board subsystems, such as Wheel Slide Protection (WSP) system and antiskid device. The integration and mutual interaction between the two systems are continuously increasing on high speed applications, especially when degraded adhesion conditions occur. Traditionally, braking and traction performances should be verified on a full-scale roller rig to avoid expensive on line tests. In this work the authors investigate a complex application in which the braking and traction systems are tested using a full scale roller rig able to perform the simulation of a known wheel-rail adhesion pattern (especially degraded adhesion) applying a HIL (Hardware In the Loop) approach. This approach is also followed by the innovative roller rig built by RFI (Rete Ferroviaria Italiana) and Trenitalia in the Research and Approval Center of Firenze-Osmannoro. The analysis is performed considering the complex interaction between vehicle on board subsystems and the test rig logics and it is validated considering experimental results available from previous research activities acquired by Trenitalia and RFI.
\end{abstract}

(c) 2013 Elsevier Ltd. All rights reserved.

\section{Introduction}

Usually, in railway vehicles, the longitudinal train dynamics is controlled by traction and braking on board subsystems. The integration and mutual interaction between the two systems are continuously increasing especially on high speed applications [1] as stated by the attention on this aspect of the European railway standards such as TSI (Technical Specification for Interoperability).

In railway applications, Hardware in the loop (HIL) systems (such as full scale roller rigs) are traditionally used to test traction and braking performances of complete railway vehicles [2]. Modern traction and braking systems are equipped with specific on board subsystems, respectively named anti-skid (for traction) and WSP (Wheel Slide Protection system, for braking); such subsystems are able to correct the longitudinal efforts applied to the axles to prevent excessive sliding between wheels and rail; it is important to limit the slidings because they may seriously damage the contact surfaces, negatively affect braking and traction performances and consequently safety, signalling and traffic management.

The simulation of degraded adhesion conditions is sometimes performed on roller rigs to identify the behavior both of the

\footnotetext{
* Corresponding author. Tel.: +39055 4796332.

E-mail addresses: benedetto.allotta@unifi.it (B. Allotta), roberto.conti@unifi. it (R. Conti), meli@mapp1.de.unifi.it (E. Meli), luca.pugi@unifi.it (L. Pugi), a.ridolfi@unifi.it (A. Ridolfi).
}

vehicle and of the friction and wear [2-4]. However the simulation testing of braking and traction subsystems on roller rigs considering degraded adhesion conditions is still limited to few applications [5], since sliding between roller and wheel produces wear of the rolling surfaces; this event is not acceptable because of the corresponding effect on maintenance costs of the rig (rollers have to be turned or substituted) and the potentially dangerous working conditions arising from the change of contact conditions due to wear.

In previous research activities [3] the authors have proposed a robust control system, analyzed only for the traction phase and especially studied for HIL architectures, able to reproduce on the rollers a virtual adhesion pattern (particularly degraded adhesion conditions) performing a simulation of mechanical impedance: roller motors are controlled in order to reproduce the same tangential efforts exchanged between the wheelsets and the rail and calculated by a reference virtual railway vehicle model. Since the real adhesion factor between the roller and the wheel is far higher than the simulated one, negligible sliding occurs between them. The main limitations of this work are the simplicity both of the $2 \mathrm{D}$ vehicle and roller-rig models and of the contact model between roller and wheel; moreover, even the wheel-rail adhesion model in the virtual railway vehicle model was very simple and not validated by experimental results.

The design and the construction of complex HIL systems such as fullscale roller rig involves high economic investments; therefore the development of scaled roller rig may allow the reduction 
of the costs. As concerns this topic, in previous works authors designed and modeled a scaled roller rig inspired to the Osmannoro fullscale roller rig [6-8].

In parallel with the HIL systems, the Software In the Loop (SIL) systems permit to produce a complete virtual model of the HIL system; in this way, all the components of the system are completely simulated. This approach is very useful to reduce the initial economic investment and, at the same time, to preliminary test the dynamic response of the HIL system in presence of several working conditions or to test different control strategies.

The innovative features of this work (compared with the previous activities) can be summarized in the following points:

1. implementation of a complete HIL simulator (SIL approach) of a 3D fullscale test rig (inspired to the Osmannoro test rig) including the hardware part, the software part, the estimator part and the controller;

2. 3D multibody models of the vehicles and of the roller-rig. The vehicle models are inspired on the E402 B and UIC-Z1 real characteristics [5];

3. 3D contact model especially studied for revolute surfaces [6] and applied in the interfaces between the wheels of the vehicle and the rollers of the roller-rig;

4. 2D adhesion model based on the energetic criterion [9] and especially developed for this application within the virtual railway vehicle model;

5. WSP and antiskid models inspired on the real device features $[10,11]$;

6. validation of the presented HIL architecture in terms of capacity to reproduce on the test rig braking and traction phases (under degraded adhesion conditions), thanks to the innovative adhesion model implemented in the virtual railway vehicle model; the validation has been performed by means of experimental data coming from several tests of traction and braking phases performed by Trenitalia under degraded adhesion conditions [11,12] with two Trenitalia vehicles, respectively E402B and UIC-Z1;

7. analysis of the controller performances (based on the controller [3] and with new improvements to reject disturbances) and of the dynamical behavior of the simulated HIL system when braking and traction phases in degraded adhesion conditions are reproduced.

In this paper the complete simulated HIL model (SIL approach) is presented. The implementation of the HIL system is inspired to the fullscale roller rig placed in the Osmannoro Research Center (built by RFI). The characteristic feature of this HIL system is the capacity to reproduce the same dynamical behavior of the railway vehicle under degraded adhesion conditions. Referring to this HIL system, the proposed architecture consists of four part: the hardware part, the software part, the estimation system and the controller. The hardware part represents the roller rig and the railway vehicle. In this paper, both the physical roller rig and the physical railway vehicle are modeled. The considered railway vehicles, modeled using a 3D multibody model are respectively the vehicle UIC-Z1, as regards the braking phase while, the locomotor E402B concerning the traction phase. Also the fullscale roller rig is simulated using a 3D multibody model. The contact between these models is performed through the innovative 3D contact model developed for revolute surfaces [6]; particular attention is also paid to the realistic WSP-antiskid models [5,12]. The software part represents the part where, using a Real-Time software, a virtual railway vehicle in different degraded adhesion conditions is simulated. This part defines the running conditions which the roller rig has to reproduce. It consists of a $2 \mathrm{D}$ simplified model of the railway vehicles where degraded adhesion conditions occur between wheels and rail. Moreover, in this part, an innovative adhesion model (based on previous research activities $[13,14]$ ) is especially developed for the HIL system. The estimation part is necessary to the HIL system to estimate the torque produced by the railway vehicle (because no sensors are installed on the vehicle) and it is referred to a previous work [3]. The estimator uses the measured roller angular speeds and the tangential contact forces on the supports to calculate the vehicle torque. The controller part is able to reproduce on the roller rig the same mechanical impedance, in terms of angular velocities and tangential contact forces, computed by the software part (where degraded adhesion conditions are reproduced) through an innovative sliding mode controller (SMC) based on previous research activities [15].

\section{General architecture of the fullscale roller rig}

The general architecture of the simulated HIL system (with hardware part, software part, estimator system and controller) is schematically shown in the block diagram of Fig. 1.

The following main blocks can be identified in the scheme:

1. Test-rig model: the test rig model, representing the models of the hardware part, consists of two different systems: the 3D railway vehicle model and the $3 \mathrm{D}$ roller rig model (based on the real characteristics of the vehicles). The 3D railway vehicle model depends on the analyzed case: in the braking case, the simulated vehicle is the UIC-Z1 while, in the traction case, the simulated vehicle is the E402B; also the WSP-antiskid models based on the real characteristics are implemented. The interactions between the wheels and the rollers is simulated using an improvement of the 3D contact model presented by the authors in [6]. The 3D roller rig model consists of a multibody model of the test rig inspired on the specifications and characteristics of the Osmannoro one.

2. Virtual railway vehicle model: it represents the model of the vehicle used to simulate the locomotive behavior on the rails in different adhesion conditions [3] (software part of the HIL scheme and designed for a real-time implementation). This 2D multibody model simulates the longitudinal dynamics of the vehicle and the $2 \mathrm{D}$ adhesion model implemented in this part presents several innovative characteristics [16] which permit to accurately approximate the real behavior of the adhesion coefficient during braking and traction phases under degraded adhesion conditions. The inputs are the estimated torques on the wheelsets and the outputs are the simulated angular velocities and the simulated tangential contact forces.

3. Controllers: the controller model should reproduce on the roller rig the same dynamical behavior of the virtual train model in terms of wheel angular velocities and vehicle motor torques $[3,15]$. Due to the HIL system non-linearities in the controller a sliding mode approach is adopted (controller). The new controller law (if compared to the previous work [3]) law has been modified to guarantee the robustness and the dynamical stability of the new whole 3D HIL system.

4. Torque estimator: the data measured by the sensors installed on the roller rig are the roller angular velocities and the longitudinal contact forces on the roller supports. This choice is mainly due to the HIL architecture (to speed up the set up process, because no sensors will be placed on the vehicle). The estimator block allows the estimation of the creep forces and of the wheel angular accelerations to calculate the estimated torques applied by the bogie motors on the wheelsets (estimator system).

The SIL approach has been implemented in the MATLAB ${ }^{\mathbb{R}}$ Simulink ${ }^{\circledR}$ environment particularly the multibody model has been implemented in the Simulink ${ }^{\circledR}$ toolbox SimMechanics. 


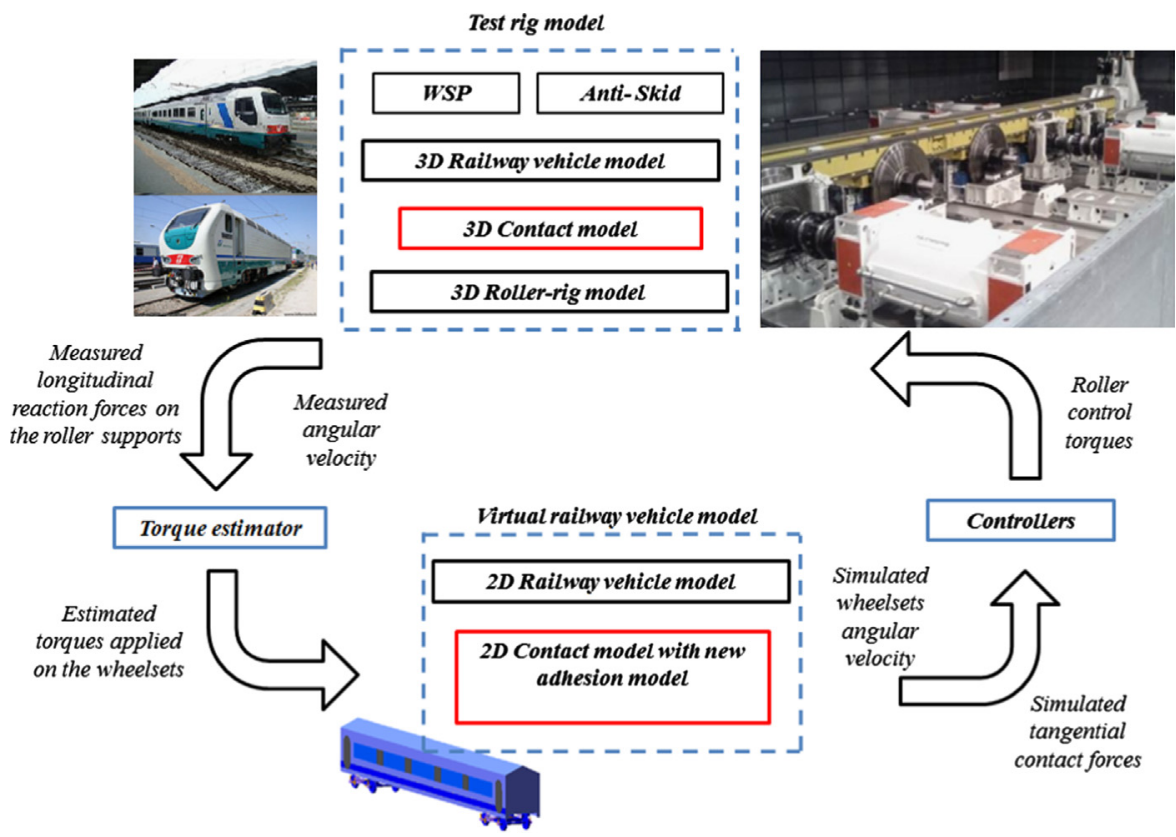

Fig. 1. General architecture of the simulated HIL system

\section{The simulated HIL system description}

The simulated HIL system (presented in Fig. 1) consists of four parts: the test rig model, the torque estimator, the virtual railway vehicle model and the controller. In this section all the components of the system will be explained in detail. The complete model of the simulated HIL system and the flow of the data between the subsystems are shown in Fig. 2.

\subsection{Test rig model}

\subsubsection{Test rig model: the vehicle model}

The railway vehicle has been modeled through a 3D multibody model using a parametric approach. The authors characterized the model in manner to simulate the vehicles used in the experimental tests. In particular the tests performed by Trenitalia involve two different vehicles: UIC-Z1 for braking tests and E402 B for traction tests.

The UIC-Z1 is a railway passenger coach, simulated by a complete 3D model with one coach connected to two bogies. A two-stage suspension system is used to link the coach to the four wheelsets. The primary and the secondary suspensions consist of a systems with linear stiffness and damping in longitudinal, vertical and lateral directions. There are also damping devices with nonlinear characteristics as anti-yaw dampers, anti roll-bar and bumpstop plugs (to reduce the undesired coach motions). A complete description of the model and the physical parameters are defined in a previous work [12]. The E402B model is a locomotor vehicle, simulated by a complete 3D model with one coach, two bogies and four wheelsets. The connections between coach, bogies and wheelsets are realized by two suspension levels (primary and secondary) and several damping devices with non-linear characteristics. Also in this case, the vehicle has a $B_{0}-B_{0}$ wheel-and-axle set. The complete vehicle description can be found in [12].

The inputs of the model are the torques $C_{s}$ modulated by the on board subsystems and the contact forces while the outputs of the model are the kinematic wheelset variables transmitted to the 3D contact model, the original torques $C$ (without the on board subsystems modulation) and wheelset angular velocities $\omega_{w}$. These last two outputs are directly measured by the on board subsystems loops and they are not accessible by the HIL system. The multibody models have been implemented in the MATLAB ${ }^{\mathbb{R}}$ toolbox SimMechanics (Fig. 3).

\subsubsection{Test rig model: the roller rig model}

The roller rig model is a 3D multibody model of the roller rig. The model has been built using a parametric approach (see Fig. 4); in this way, different test rig can be studied. In particular, the authors focused on the characterization of the roller rig inspired to the Osmannoro roller rig (installed in the Osmannoro Research Center). The model consists of four rollers with a particular roller profile able to exactly reproduce the UIC60 rail pattern with different laying angle. The test rig is simulated through rigid rollers controlled by direct drive motors (the motor dynamics is neglected); the vehicle is axially constrained on the rollers using an axial link modeled by means of a force element with a linear stiffness and damping.

The inputs of the test rig model are the torques $u$ evaluated by the controllers in order to reproduce on the test rig the same dynamical behavior of the virtual railway model. The outputs of the model, inspired on the Osmannoro roller rig architecture, are the roller angular velocities $\omega_{r m}$ and the tangential component of the contact forces $T_{m i s}$ measured on the roller supports.

\subsubsection{Test-rig model: $3 D$ contact model}

The 3D contact model represents an improvement compared to previous works of the authors [6,4] and it is based on a semianalytical method previously developed by the authors for the contact between wheels and rail [13]. This contact model has been especially designed to allow the contact points detection between two generic revolute surfaces and the calculation of the normal and tangential contact forces. In literature different approaches are presented [17-19] to find the contact points. Those adopted in the roller rig simulator are based on a semi-analytical procedure $[20,21]$ which satisfies the following statements:

1. generic wheel-roller profiles;

2. the contact detection algorithm between revolute surfaces is fully 3D and does not introduce simplifying assumptions on the problem geometry and kinematics; 


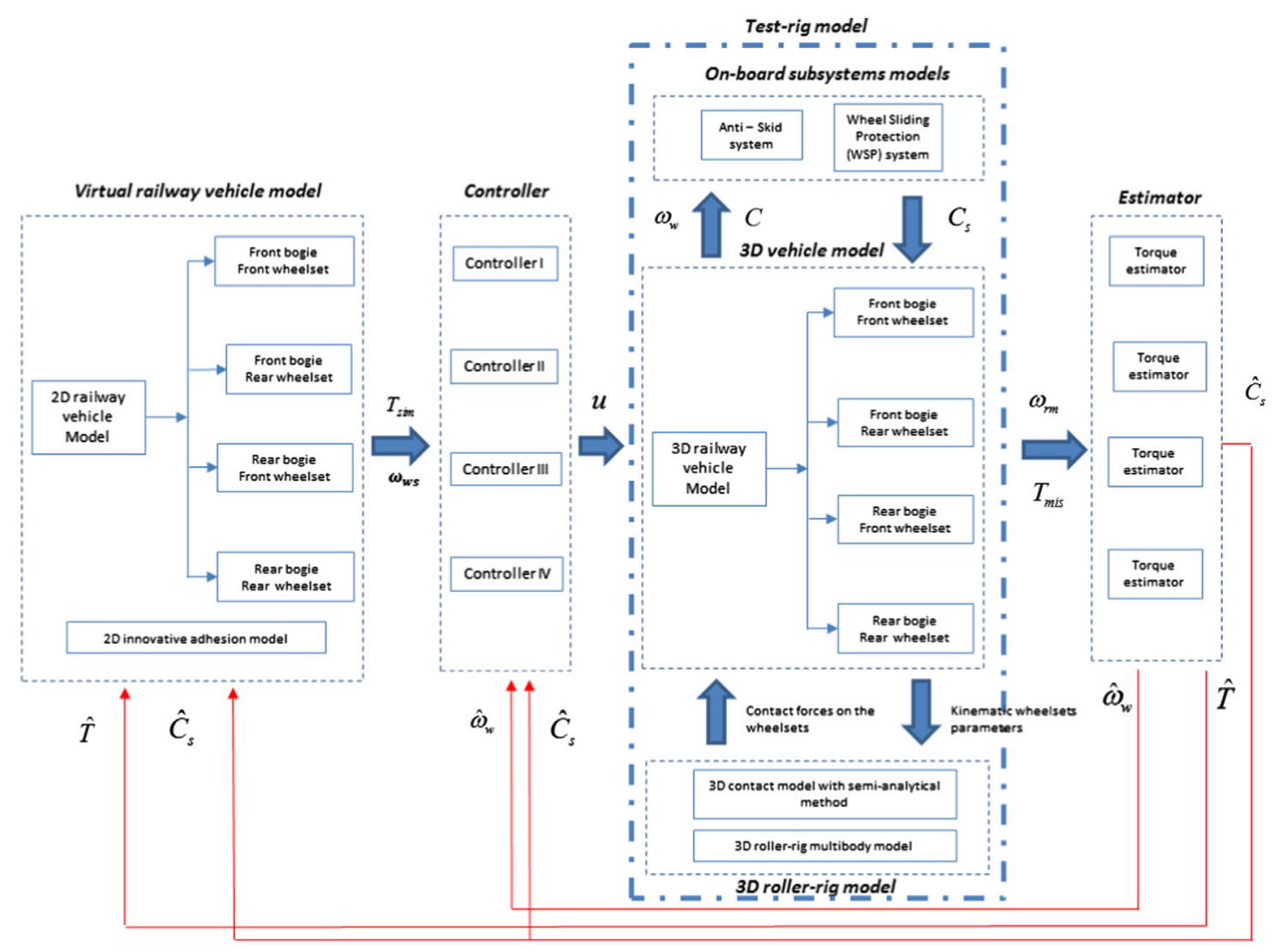

Fig. 2. Detailed scheme of the simulated HIL system.

3. no limits concerning the number of multiple contact points;

4. high computational efficiency needed for the online implementation of the algorithm within multibody models without employing discretized look-up table.

Contact point detection: the new procedure for the contact point detection starts from the standard idea that the contact points between the wheel surface and the roller surface are observed where the distance between the two surfaces assumes a stationary point and it is based on the parallelism conditions among the normal unity vectors of the wheelset and of the roller surfaces:

$\mathbf{n}_{r}^{r}\left(\mathbf{p}_{r}^{r}\right) \| \mathbf{n}_{w}^{r}\left(\mathbf{p}_{w}^{r}\right) \rightarrow \mathbf{n}_{r}^{r}\left(\mathbf{p}_{r}^{r}\right) \times[\mathbf{R}] \mathbf{n}_{w}^{w}\left(\mathbf{p}_{w}^{w}\right)=\mathbf{0}$

$\mathbf{n}_{r}^{r}\left(\mathbf{p}_{r}^{r}\right) \| \mathbf{d}^{r} \rightarrow \mathbf{n}_{r}^{r}\left(\mathbf{p}_{r}^{r}\right) \times[\mathbf{R}] \mathbf{d}^{r}=\mathbf{0}$

where $\mathbf{n}_{\mathbf{r}}^{\mathbf{r}}$ and $\mathbf{n}_{\mathbf{w}}^{\mathbf{w}}$ are the normal vectors of the wheelset and roller surfaces referred to the roller and to the wheelset reference frame respectively while $\mathbf{d}^{\mathbf{r}}$ is the distance vector expressed in the roller reference frame. The original $4 \mathrm{D}$ system can be analytically reduced to one single scalar equation $F\left(y_{w}\right)=0$ (that, at this point, can be easily solved numerically) by expressing the variables $x_{w}, x_{r}$, $y_{r}$ as a function of $y_{w}$. In particular the considered analytical procedure does not require any simplifying kinematical or geometrical hypothesis and therefore does not involve any loss of generality. The solution of this analytical procedure (see [6] for the complete procedure) leads to the following 1D scalar equation where the unique unknown is $y_{w}$ :

$$
\begin{aligned}
F_{1,2}\left(y_{w}\right)= & -r^{\prime}\left(y_{r 1,2}\right) r\left(y_{r 1,2}\right)\left(G_{z}+y_{w} r_{32}-r_{33} \sqrt{w\left(y_{w}\right)^{2}-x_{w 1,2}^{2}}-\sqrt{r\left(y_{r}\right)^{2}-x_{r}^{2}}\right) \\
& -\sqrt{r\left(y_{r 1,2}\right)^{2}-x_{r}^{2}}\left(G_{x}+x_{w 1,2} r_{11}+y_{w} r_{12}-r_{13} \sqrt{w\left(y_{w}\right)^{2}-x_{w 1,2}^{2}}-y_{r}\right)=0 .
\end{aligned}
$$

The reduction of the algebraic problem dimension (from 4D to 1D) represents the most innovative feature of the algorithm; the main benefits of the new approach are:

- high computational efficiency;

- easy management of the multiple solutions;

- also simplified algorithm (like the grid method) can be numerically efficient if applied to a scalar problem.

Adhesion model: for each contact point, individuated by the previous algorithm, it is necessary to compute the forces and the torque applied on the wheelset. The procedure used in this work consists in two different steps: the normal problem and the tangential one. The normal contact problem has been solved according to the Hertz theory while the tangential contact forces and the spin moment have been calculated by means of the saturated Kalker theory [17], in order to take into account the adhesion limit.

\subsubsection{Test rig model: vehicle on board subsystem models}

The vehicle on board subsystems are related to the safety devices installed on the railway vehicle to prevent dangerous situations. In particular, the on board subsystems, such as anti-skid device during the traction phase or Wheel Slide Protection system during the braking phase, are necessary when degraded adhesion conditions occur. These systems equip the simulated vehicles with the aim to avoid macro-sliding between rail and wheels. In both the studied cases the devices have to modulate the torques (positive in the traction phase and negative in the braking one) applied to the wheels to not exceed the adhesion limit. The architecture presented in [3] allows the simulation of the degraded adhesion conditions and this feature results quite useful 
a

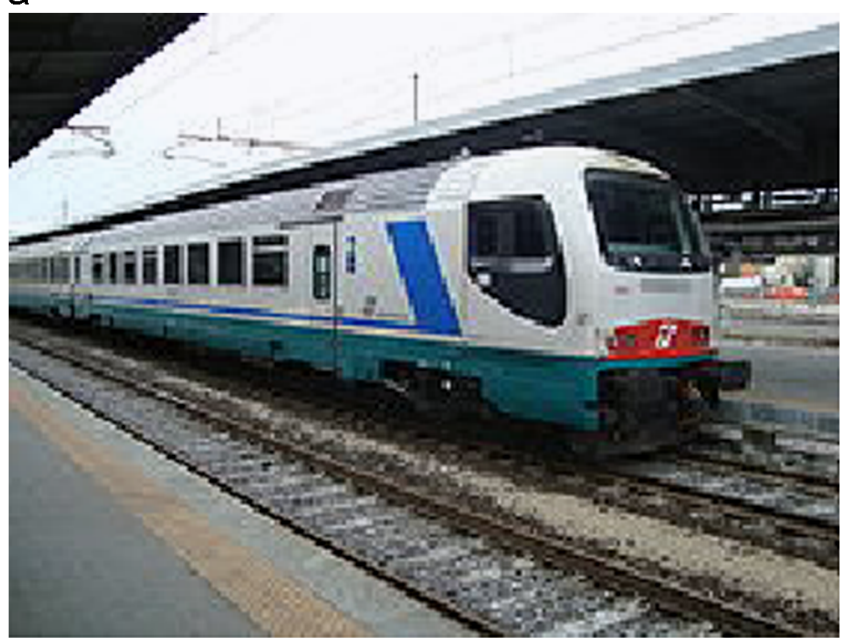

b

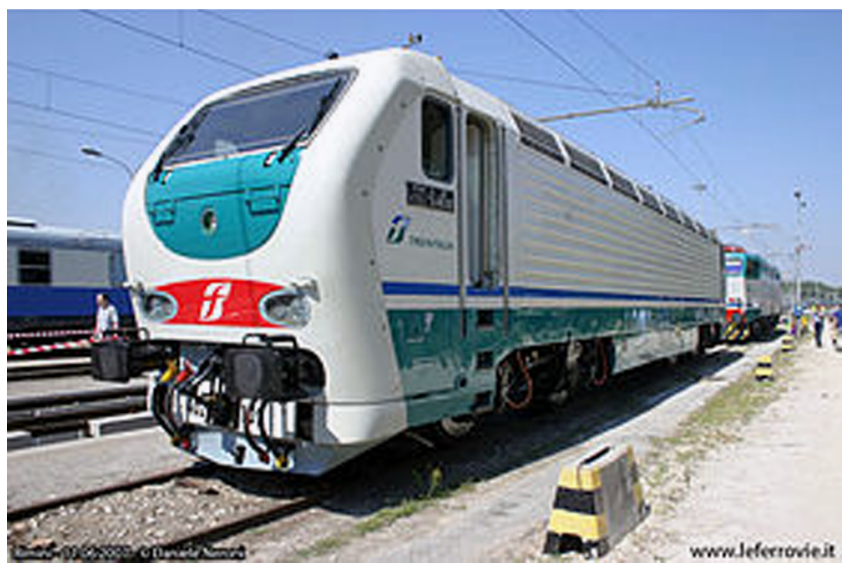

Fig. 3. UIC-Z1 (a) and E402B (b) pictures.

in the testing activity of the safety on board subsystems on the test rig; therefore, in order to correctly analyze the dynamical behavior of the HIL system, a proper modeling of the on board subsystems of the railway vehicle is necessary. The validation of this HIL architecture is performed through the experimental data (presented in Chapter 4) and related to several braking and traction tests (under degraded adhesion conditions) in which the effects of anti-skid and WSP interventions are clearly visible.

Antiskid device: the anti-skid device prevents macro-sliding during the traction phase when the torque produced overcomes the adhesion limit. This effect implies the modulation of the torque using different strategies. The simulated anti-skid logics is explained in Fig. 5 and is based on the E402B technical documentation [11] and modeled in [12].

The inputs of the anti-skid device are the four angular axle speeds $\omega_{w i}$ and the desired torques $C$ (defined by the driver maneuver). The outputs of the block are the four modulated torques $C_{s i}$. The device logic consists of three different tasks:

1. Reference evaluation: evaluation of the reference linear velocity $V_{r e f}$ and acceleration $a_{r e f}$ of the vehicle based on the wheelset angular velocities $\omega_{w i}$ and acceleration $\dot{\omega}_{w i}$;

2. Sliding state evaluation state $_{A S}$ : through a speed criterion and an accelerometer one, the logical variable state $_{A S}$ is set equal to (1) if the vehicle is sliding and equal to (0) if it is not sliding;

3. Torque modulation: in this, the torque modulation strategy to limit the sliding condition is defined.

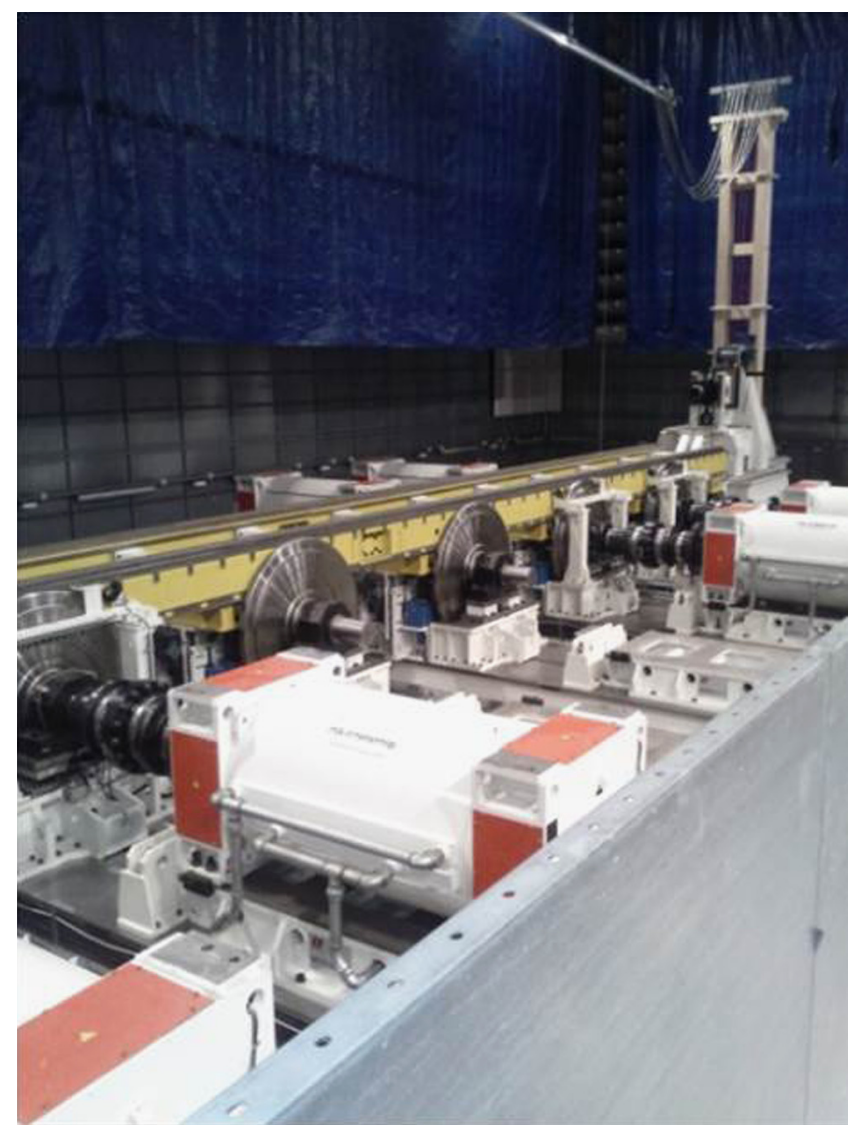

Fig. 4. Osmannoro test rig facility in the anechoic room.

Wheel Slide Protection (WSP) device: the WSP device allows the control of the applied torques to the wheels in order to prevent macro-sliding during the braking phase. The simulated model is inspired to the WSP device installed on the vehicle UIC-Z1 [10,22] and it is based on the architecture described in a previous work [12]. As can be seen in Fig. 6 the inputs of this block, which contains the WSP logics and the pneumatic braking plant, are the braking torque $C$ and the four wheelset velocities $\omega_{\text {wi }}$. The outputs are the modulated braking torques $C_{s}$. Therefore, the WSP system is closely related to the pneumatic braking plant and a proper modeling of both systems is necessary to correctly simulate the braking phase. In this paper, the authors do not describe the pneumatic braking plant model; for a detailed description [9] can be seen.

As can be observed from Fig. 6, the WSP system working principle can be divided into three different tasks:

1. Reference evaluation: evaluation of the reference linear velocity $V_{\text {ref }}$ and acceleration $a_{\text {ref }}$ based on the wheelset angular velocities $\omega_{w i}$ and acceleration $\dot{\omega}_{w i}$;

2. Sliding state evaluation: the logical state state $_{W S P}$ defines if the vehicle is sliding or not. The logical state is obtained employing a speed criterion and an accelerometer one by means of a logic table called discrete state control logics [9];

3. Periodic braking release: this technique sets the braking torque to zero on a particular wheelset with the aim of increasing the perceived adhesion coefficient $\mu$ to the original adhesion value; this approach is often used when degraded adhesion condition is very persistent and the WSP logic tends to drift.

\subsection{Virtual railway vehicle model}

The virtual railway vehicle model simulates the dynamical behavior of a vehicle during a braking or a traction phase 


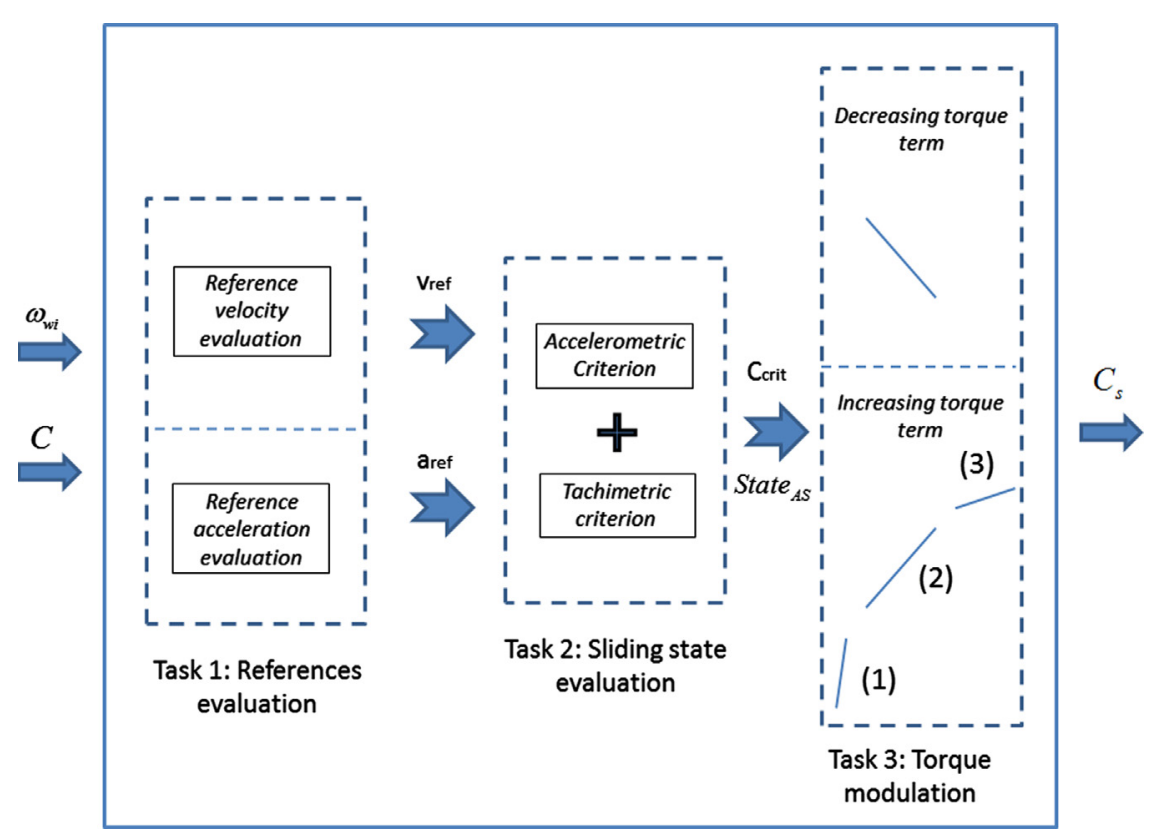

Fig. 5. Detailed architecture of the antiskid device.

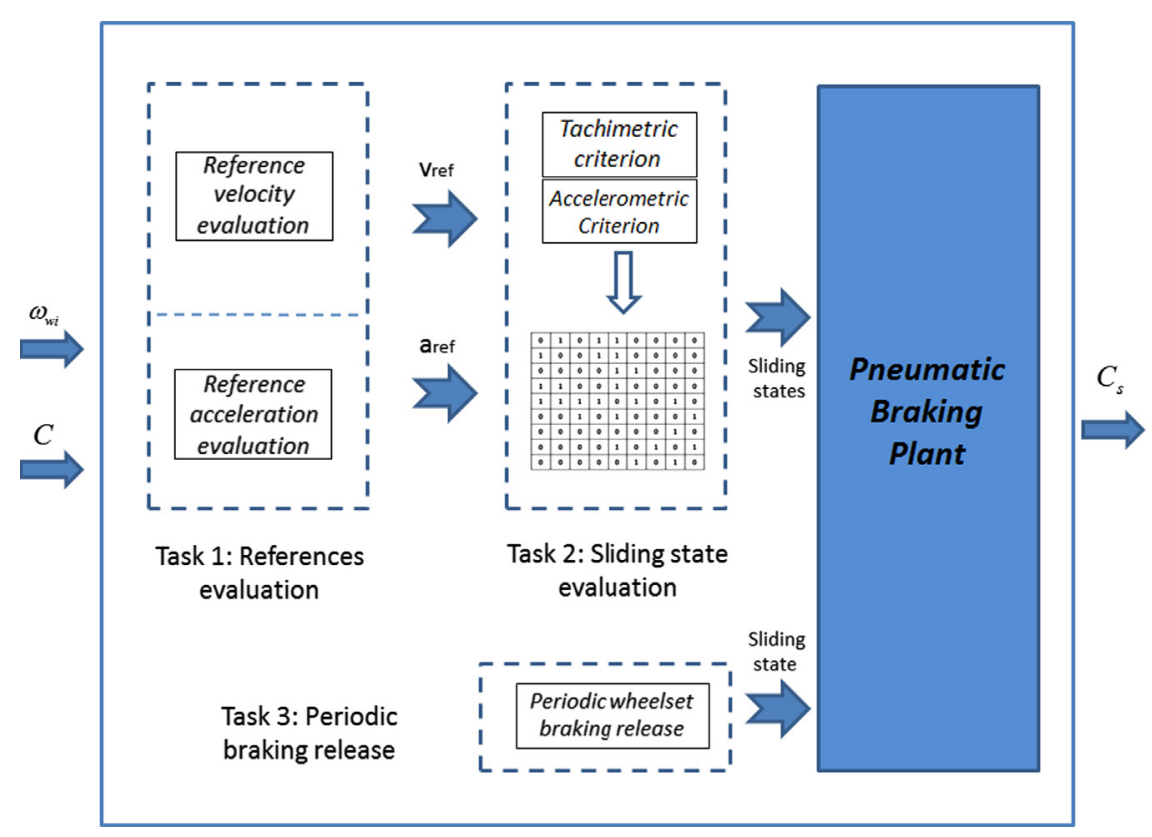

Fig. 6. Wheel Slide Protection device: logical architecture.

(respectively a UIC-Z1 and an E402B), under degraded adhesion conditions. The aim of this model is to reproduce the same dynamical behavior of the experimental tests presented in Chapter 4. The simulated degraded adhesion conditions (both in traction and in braking phases) are referred to a well defined operative conditions, usually defined by international technical rules, in terms of adhesion coefficient, vehicle characteristics, etc. According to the architecture presented in Fig. 2 the inputs of the model are the tangential contact forces $\hat{T}_{i}$ measured on the roller supports and the estimated torques $\hat{C}_{s}$. The outputs are the simulated tangential contact forces $T_{\text {sim }}$ and the simulated wheel angular velocities $\omega_{w s}$. In Fig. 7, the two main parts of the model are displayed: the vehicle model and the adhesion one.

\subsection{1. $2 D$ vehicle model}

The $2 \mathrm{D}$ vehicle model is a simplified $2 \mathrm{D}$ multibody model of the longitudinal train dynamics where the wheelset load distributions $N_{i}$ are calculated. The model consists of a carbody, two bogies and four wheelsets held by two suspensions level (primary and secondary) in which the physical parameters characterize the two vehicle models. Referring to Fig. 7, the multibody model is divided into two parts: the coach model with the coach and two bogies and the wheelset model with the wheel/rail contact model. This particular architecture permits to use the estimated tangential contact forces $\hat{T}$ in the wheelset models too; in this way the accuracy of the model can be increased. The coach model inputs are the estimated tangential forces $\hat{T}$ (estimated by the estimator 


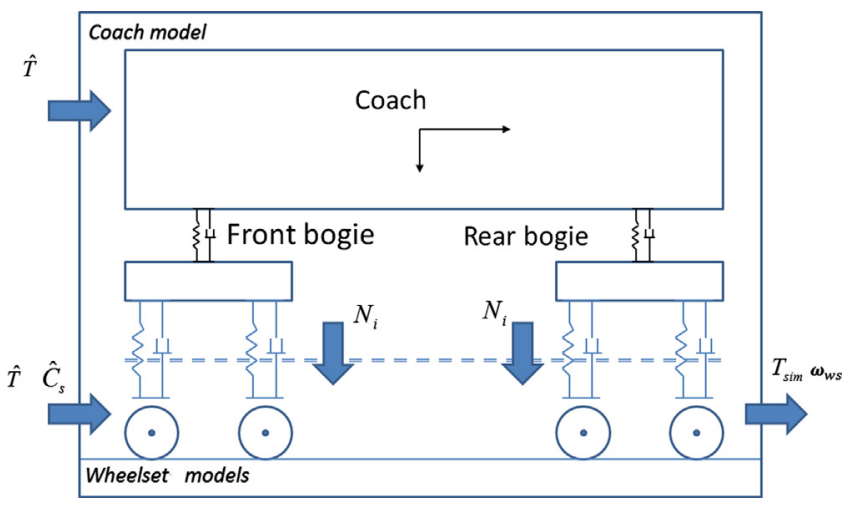

Fig. 7. Scheme of the virtual railway vehicle model.

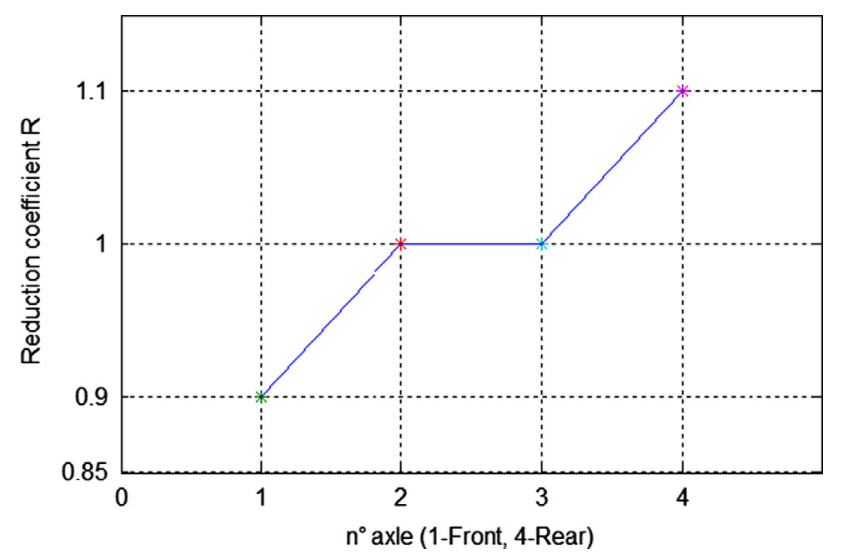

Fig. 8. Reduction parameter $R$ trend referred to the axle position along the vehicle.

block and measured from a load cell on the roller rig support) and the outputs are the normal loads $N_{i}$. The wheelset model inputs are the estimated tangential forces $\hat{T}$, the estimated torque $\hat{C}_{s}$ and normal loads $N_{i}$ coming from the coach model. The outputs are the reference values of the wheel angular velocity $\omega_{w s}$ and the simulated tangential force $T_{\text {sim }}$ evaluated by the $2 \mathrm{D}$ wheel/rail contact model.

\subsection{2. $2 D$ adhesion model}

The innovative adhesion model, presented by the authors in previous work [16], is characterized by a particular adhesion law especially designed to reproduce the adhesion coefficient trend observed in several degraded adhesion experimental tests. Some previous studies $[18,16]$ reveal hysteresis phenomena in the cycles of adhesion loss and recovery; moreover the adhesion coefficient tends to modify his behavior according to the axle kinematic energy dissipation. The proposed adhesion model simulates the dependence of the value of the available adhesion on the level of dissipated energy in the wheel-rail interface. According to the HIL architecture (Fig. 2), the simulated tangential components of the contact forces $T_{\text {sim }}$ is eventually calculated.

In order to analyze the proposed model two hypotheses are used: the passage of the following axles upon the rail provokes the clearance of a portion of the contaminating agents (according to the concept [23] that in a benchmark test with the same degraded adhesion condition on the rail, the front wheelset-front bogie has a worse degraded adhesion coefficient compared to the rear wheelset-rear bogie adhesion coefficient, see Fig. 8; this effect is amplified by the longitudinal load movement due to traction or braking phases) and a single contact point between wheel and rail is considered. The adhesion coefficient can be reduced by means of the reduction parameter $R$ as a function of the axle position $\mu_{c i}=R_{i} \mu_{c}$.

To increase the accuracy of the simulated adhesion model as regards the dissipated energy, the implemented model defines two different analytical modeling:

- Low energy dissipation: the adhesion is based on the saturated Kalker theory for the tangential problem. According to the theory, the forces are saturated using a coefficient $K_{s}$ :

$K_{S}= \begin{cases}\frac{1}{A_{S}}\left[A_{S}-\frac{1}{3}\left(A_{S}\right)^{2}+\frac{1}{27}\left(A_{S}\right)^{3}\right] & \text { for } F_{R} \leq 3 \mu_{c} N \\ \frac{1}{A_{S}} & \text { for } F_{R}>3 \mu_{c} N\end{cases}$

where $A_{S}=F_{R} / \mu_{c} N, \mu_{c}$ is the wheel-rail kinematic friction factor, $N$ is the normal force and $F_{R}=\sqrt{F_{x}^{2}+F_{y}^{2}}$ is the total creep force (where $F_{x}$ and $F_{y}$ are the longitudinal contact forces and the lateral ones). In this case, the energy dissipation in presence of variations of the relative sliding probably does not have time to modify the presence of the contaminating agents. The saturated creep forces $F_{x}^{\prime}=K_{s} F_{x}$ and $F_{y}^{\prime}=K_{s} F_{y}$ have then to be corrected using a multiplicative factor $K_{a}(\varepsilon, \dot{\varepsilon})$. This correction factor $K_{a}(\varepsilon)$, which realizes the transition between static and kinematical friction factor, is defined as a function of the relative creepage:

$F^{\prime \prime}{ }_{x}=K_{a} F^{\prime}{ }_{x}=K_{a} K_{s} F_{x}$

$F^{\prime \prime} y=K_{a} F^{\prime}{ }^{\prime}=K_{a} K_{s} F_{y}$

$T_{\text {sim }}=F_{R}^{\prime \prime}=\sqrt{\left(F^{\prime \prime}\right)^{2}+\left(F_{y}^{\prime \prime}\right)^{2}}$

Moreover, to reproduce the hysteretic behavior of adhesion, different values of $K_{s}$ coefficients are introduced as concerns the adhesion loss ( $K_{s u}$, if the relative slidings increase) and the adhesion recover ( $K_{s d}$, if the relative slidings decrease); as can be seen in Fig. 9, they are defined as

$K_{s u}(\alpha)=\left\{\begin{array}{l}1-\frac{1}{3} \alpha+\frac{1}{27} \alpha^{2}=f(\alpha) \\ \alpha \leq \alpha_{\lim }=3 \\ \frac{1}{\alpha} \\ \alpha>\alpha_{\lim }\end{array}\right.$

$K_{s d}(\alpha)=\left\{\begin{array}{l}g(\alpha) \\ \alpha \leq \alpha_{\lim }=3 \\ \frac{1}{\alpha} \\ \alpha>\alpha_{\lim }\end{array}\right.$

where $\alpha=F_{R} / \mu_{C} N, \alpha_{\text {lim }}=3$ and $g(\alpha)$ is a cubic function satisfying the following conditions: $g(0)=1, g\left(\alpha_{\lim }\right)=1 / 3, g^{\prime}(0)=-2 / 3$ and $g^{\prime}\left(\alpha_{\text {lim }}\right)=-1 / 9$. The dependence from creep time derivative has

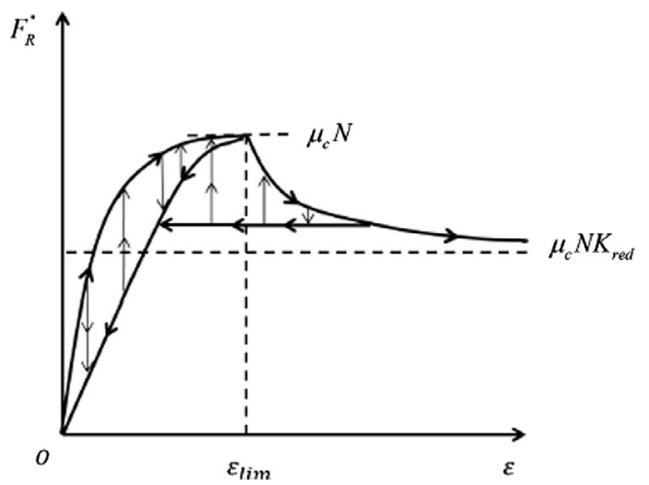

Fig. 9. Hysteretic cycle of tangential forces in a cycle of adhesion loss and recovery. 
been added to simulate the hysteretic cycle of the adhesion factor $K_{a}(\varepsilon, \dot{\varepsilon})$ :

$$
\begin{aligned}
& K_{a}^{*}= \begin{cases}K_{a}^{*}=1 \\
K_{a}^{*}=K_{r e d}+\left(1-K_{r e d}\right)^{-\lambda\left(\varepsilon-\varepsilon_{\lim }\right)} & \varepsilon>\varepsilon_{\text {lim }}\end{cases} \\
& \dot{\varepsilon} \geq 0 \Rightarrow \begin{cases}K_{a}=K_{a}^{*}(\varepsilon) \\
K_{s}=K_{s u}(\alpha)\end{cases} \\
& \dot{\varepsilon}<0 \Rightarrow \begin{cases}\tilde{K}_{a}=K_{a}^{*}(\tilde{\varepsilon}) \\
\tilde{K}_{s}=K_{s d}(\tilde{\alpha}) \\
\text { if } \tilde{\varepsilon} \leq \varepsilon_{\lim } & K_{a}=K_{a}^{*}(\varepsilon) \\
\text { if } \tilde{\varepsilon}>\varepsilon_{\lim } & K_{s}=K_{s d}(\alpha) \\
\text { if } K_{s d}(\alpha) K_{a}^{*}(\varepsilon) \leq \tilde{K}_{a} \tilde{K}_{s}\end{cases} \\
& K_{a}=K_{a}^{*}(\varepsilon) \\
& K_{s}=K_{s d}(\alpha)
\end{aligned}
$$

where with " " are indicated the memorized values corresponding to the adhesion inversion instant from $0^{+}$to $0^{-}, \varepsilon_{\text {lim }}$ is the $\varepsilon$ value corresponding to $F_{R}\left(\varepsilon_{\text {lim }}\right)=3 \mu_{c} N$ (the $\varepsilon$ value corresponding to the maximum value of the adhesion coefficient $\mu^{*}$ ), $K_{\text {red }}=0.65$ is the kinematical friction reduction factor, $\lambda=50$ is the exponential link slope factor.

The adhesion coefficient computed in this case, which is a function of the relative sliding $\varepsilon$ and its time derivative $\dot{\varepsilon}$, is defined as:

$\mu^{*}=\mu^{*}(\varepsilon, \dot{\varepsilon})=\frac{F^{\prime \prime}}{N}=\frac{T_{\text {sim }}}{N}$.

- High energy dissipation: in this case the energy dissipation linked to relative slidings $\varepsilon$ may become considerable and probably cause the progressive removal of the contaminating agents in the wheel-rail interface. The adhesion law takes into account the sliding $\varepsilon_{\text {wspec }}$ and the associated specific sliding power:

$\varepsilon_{\text {wspec }}=\frac{\left|\underline{V}-\underline{\omega_{w}} \wedge(G-C)\right|}{\left|\underline{\omega_{w}} \wedge(G-C)\right|}$

$W_{\text {wspec }}(t)=\mu_{n f}(t) N \varepsilon_{\text {wspec }}$

where $\mu_{n f}$ is the adhesion coefficient defined by (12) and $N$ is the vertical load. The wear of the sliding surface is proportional to the corresponding specific power; when the specific power is

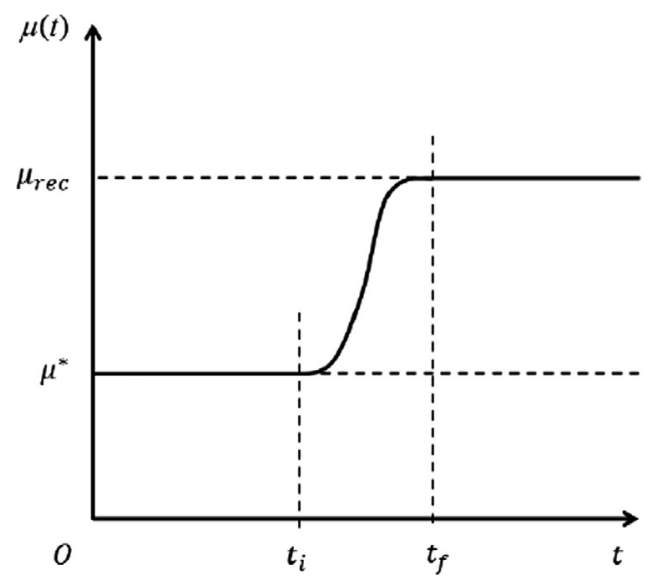

Fig. 10. Transition of the friction coefficient following up wear phenomena $\left(t_{f}-t_{i}=0.4 \mathrm{~s}\right)$. higher than a certain value, the amount of energy is enough to destroy the contaminating agents (bringing back the elevated adhesion level). The problem is estimating the value of the specific power to produce this effect. The authors propose to utilize some basic quantities defined in the rule [22]. Referring to this rule and to a previous work [16,24], the specific power, which probably completely remove the contaminating agents, is estimated in $7800 \mathrm{~J} / \mathrm{m}$ applied for $0.4 \mathrm{~s}$.

As a qualitative reference in Fig. 10 is shown the trend of the friction coefficient time dependence due to wear phenomena. The test described in the article [24] is a pin-on-disc type with a contaminating agent film inserted between them: the results point out a transition (rather quick) of the friction coefficient, caused by the wear linked to the sliding of the surfaces, which leads to a more elevated friction level (corresponding to the condition of contaminating agent completely removed).

Therefore the authors have decided to use a second order transfer function $t f$ reproducing, with a good approximation, this type of behavior

$t f(s)=\frac{1}{\tau^{2} s^{2}+2 \xi \tau s+1}$.

Supposing consequently the adhesion level linearly connected to the material removal, a new adhesion law is proposed (12):

$\mu_{n f}(t+\Delta t)= \begin{cases}\mu^{*}(t) & \text { if } W_{\text {wspec }}<W_{\text {lim }} \\ K_{W}(t)\left(\mu_{\text {rec }}-\mu^{*}(t)\right)+\mu^{*}(t) & \text { if } W_{\text {lim }} \leq W_{\text {wspec }} \\ \mu_{\text {rec }} & \text { if } W_{\text {wspec }}>W_{\max }\end{cases}$

where

1. $K_{W}(t)$ is a function of the specific sliding $K_{W}(t)=\left(W_{\text {wspec }}(t)-W_{\text {lim }}\right) /\left(W_{\max }-W_{\lim }\right)$;

2. $\mu^{*}$ is the adhesion law (8). Inside this law, $\mu_{c}$ is the maximum value of the adhesion coefficient (corresponding to $\varepsilon=\varepsilon_{\text {lim }}$ ), defined equal to 0.08 in the simulations of degraded adhesion braking tests and 0.07 in degraded adhesion traction tests;

3. $\mu_{\text {rec }}$ is equal to the effective maximum value of available adhesion, if we consider its recovery as a consequence of the dissipation of energy $\left(\mu_{\text {rec }}=0.10\right.$ in braking tests and $\mu_{\text {rec }}=0.09$ in traction tests);

4. $W_{\max }$ is the value of specific sliding power the complete recovery of adhesion corresponds to. This value is estimated (under the above rule [22]) equal to $7.8 \mathrm{~kJ} / \mathrm{m}$;

5. $W_{\text {lim }}$ is the specific dissipated power which can be indefinitely tolerated without causing damage to the sliding surfaces $\left(W_{\text {lim }}=1.6 \mathrm{~kJ} / \mathrm{m}\right.$ for both braking and traction tests).

The parameters $\mu_{c}, \mu_{\text {rec }}$ and $W_{\text {lim }}$ represent the tuning quantities to obtain the experimental behavior. However, it has to be noticed that, according to the new adhesion model, the experimental friction factor $\mu_{s p}^{c}$ must be approximately equal to the product between the simulated friction factor $\mu_{c}$ and the friction reduction factor $K_{\text {red }}$.

To update the $\mu^{*}$ value at each instant of time, a discrete transfer function $H(z)$ has been implemented, corresponding to the continuous one $t f(11)$, i.e. an appropriate transfer function able to reproduce the experimental trend of the friction coefficient seen in Fig. 10. The discrete conversion by Tustin bilinear transform is

$H(z)=t f\left(\frac{2(z-1)}{\Delta t(z+1)}\right)$

$H(z)$ is the appropriate transfer function able to reproduce the experimental trend of the friction coefficient

$\mu(t+\Delta t)=H(z) \mu_{n f}(t+\Delta t)$. 
The results of this work have been achieved implementing $t f$ with $\xi=1$ (to prevent unwelcome overshoots) and $\tau=0.1 \mathrm{~s}$. The numerical values inside $t f$ have been implemented considering that the time needed for the complete removal of the contaminating agent between wheel and rail is equal to $0.4 \mathrm{~s}$. Through (14) the adhesion coefficient is linked to the power dissipated by the wheel-rail slidings:

$\mu=\mu\left(\varepsilon, \dot{\varepsilon}, W_{\text {wspec }}\right)$.

\subsection{Controller}

The controller target is to be able to reproduce on the roller rig the dynamical behavior of the virtual railway vehicle in terms of angular velocities $\omega_{w}$ and torque $C_{s}$. The controller architecture was widely explained in previous works $[3,6]$. The inputs of the controller are the simulated tangential forces $T_{\text {sim }}$, the simulated wheel angular velocities $\omega_{w s}$, the estimated wheel angular velocities $\hat{\omega}_{w}$ and the estimated motor torques $\hat{C}_{S}$. The outputs are the four control roller torques $u_{i}$ (see Fig. 2). The controller layout consists of four independent controller for each roller, in order to reproduce different adhesion conditions. The controller strategy consists in a sliding mode control based on the dynamical equations of the roller rig, in this way, it is possible to reduce the disturbance effects in terms of non-linearity of the system and physical parameter uncertainties. The total control torque is defined as

$u_{\text {tot }}=u_{\text {cont }}+u_{\text {disc }}$

where:

- $u_{\text {cont }}$ is the control part based on the dynamic model:

$u_{\text {cont }}=\frac{R}{r_{v i}}\left(\hat{C}_{S}\left(1-\frac{J_{B}}{J_{v i}}\right)+\frac{J_{B}}{J_{v i}} T_{\text {sim }} r_{v i}\right)$,

where $J_{B}$ is the total moment of inertia of the roller and the simulated vehicle defined considering the wheel rotation axis:

$J_{B}=\left(J_{v i}+J\left(\frac{r_{v i}}{R}\right)^{2}\right)$

where $R$ is the roller radius, $r_{v i}$ is the wheel radius (depending of the vehicle model), $J_{v i}$ is the moment of inertia of the wheelset, $J$ is the roller inertia moment (see Table 1);

- $u_{\text {disc }}$ is the control part related to the rejection of the parameter uncertainties:

$u_{\text {disc }}=k \operatorname{sign}\left(\omega_{w s}-\hat{\omega}_{w}\right)$,

where $\omega_{w s}$ represents the simulated angular velocity and $\hat{\omega}_{w}$ is the estimated angular velocity.

The controller performances define the tendency of the control strategy to tracking the desired behavior and they are evaluated by means of two parameters:

- speed error $e_{\omega}$ : error between the simulated wheel angular velocity $\omega_{w s}$ and the estimated wheel angular velocity $\hat{\omega}_{w}$

Table 1

Test rig characteristics.

\begin{tabular}{lll}
\hline Characteristic & Parameter & Value \\
\hline Roller radius & $r$ & $0.75 \mathrm{~m}$ \\
Roller rotational inertia & $J$ & $520 \mathrm{~kg} \mathrm{~m}^{2}$ \\
UIC-Z1 wheel radius & $r_{v 1}$ & $0.445 \mathrm{~m}$ \\
UIC-Z1 rotational inertia & $J_{v 1}$ & $160 \mathrm{~kg} \mathrm{~m}^{2}$ \\
E402 B wheel radius & $r_{v 2}$ & $0.625 \mathrm{~m}^{2}$ \\
E402 B rotational inertia & $J_{v 2}$ & $374 \mathrm{~kg} \mathrm{~m}^{2}$ \\
\hline
\end{tabular}

torque estimation error $e_{c}$ : error between the real torque $C_{s}$ and the estimated torque $\hat{C}_{s}$.

\subsection{Estimator}

According to the HIL architecture (inspired by the Osmannoro one [3]), the torque applied by the wheel motor to the roller is a function of the behavior of the virtual railway vehicle model. The problem is that the vehicle torque cannot be directly measured because the proposed roller rig architecture reduces the number of sensors mounted on the vehicle.

The measurable outputs of the roller rig by means of the sensors are the roller angular velocities $\omega_{r m}$ and longitudinal component $T_{m i s}$ of the reaction force evaluated on the rollers support. Therefore, the objective of the estimator block is to estimate the wheel angular velocity $\hat{\omega}_{w}$, the wheel angular acceleration $\hat{\dot{\omega}}_{w}$, the tangential component of the contact forces $\hat{T}$ and the torque of the wheel motor $\hat{C}_{s}$ (see Fig. 2) starting from such quantities. In the HIL proposed strategy there is one estimator block for each roller. The inputs of the estimator blocks are the roller angular velocities $\omega_{r m}$ and the tangential component $T_{m i s}$ of the contact force evaluated on the roller supports. The outputs of the estimator blocks are the estimations of the wheel angular velocity $\hat{\omega}_{w}$, the tangential component of the contact forces $\hat{T}$ and the vehicle torque $\hat{C}_{s}$ applied on the wheelsets. In the roller rig the adhesion conditions between wheel and roller are good (the adhesion coefficient is $\mu_{k i n}=0.3$ ). Since in adhesion state the slidings between wheel and roller can be neglected, the following estimations hold:

$\hat{\omega}_{w}=-\frac{R}{r_{v i}} \omega_{r m}, \quad \hat{T}=T_{m i s}, \quad \hat{\dot{\omega}}_{w}=-\frac{R}{r_{v i}} \frac{d}{d t} \omega_{r m} ;$

where $\hat{\omega}_{w}, \hat{T}, \hat{\dot{\omega}}_{w}$ are the estimations of the considered quantities. The derivative operation $(d / d t) \omega_{r m}$ has to be robust taking into account the numerical noise affecting $\omega_{r m}$. At this point, to estimate the wheel motor torque the estimator blocks use a simplified dynamical model of the wheel-roller system:

$\hat{C}_{s}=\hat{T} r_{v i}+J_{B} \hat{\dot{\omega}}_{w}$

where $r_{v i}$ is the wheel radius (depending on the vehicle), $J_{B}$ is the total momentum of inertia of the axle/roller system calculated with respect of wheel rotation axis.

\section{Experimental data: braking and traction tests}

In this section the authors present the experimental data used as reference in this article. The experimental data are referred to several braking and traction tests operated on the railway line by Trenitalia. These tests have been used to test the efficiency of the safety on board subsystems (Wheel Slide Protection for braking phase and antiskid device for traction phase) and they are ruled by international technical standards $[10,22]$. The main objective of this paper is to test and to analyze the HIL proposed architecture when degraded adhesion condition are simulated in manner to evaluate if the response of the railway vehicles are the same of the experimental tests. Therefore, as previously said in the introduction, the performance of the HIL system will be evaluated in terms of HIL system performances and controller performances. The HIL system performances are related to the HIL system capacity to simulate on the test rig the same degraded adhesion conditions measured in the experimental tests. The comparison between experimental and numerical test is performed both qualitatively and using statistical indexes. The controller performances indicate the efficiency of the controller to reproduce the informations coming from the virtual railway vehicle model (analyzed only in a qualitatively way). 


\subsection{Statistical indexes}

The main statistical indexes used in this paper allow the evaluation of the HIL system performances since they characterize the velocity trends of different experimental tests in a general way. Indeed, the large use of statistical indexes is widely related to the nature of the simulated phenomena. Degraded adhesion conditions are quite complex phenomena to simulate and even experimental acquisition referred to a single nominal test usually produce several different data. This effect depends on both the intrinsic characteristic of the simulated phenomena and the on board subsystem behaviors that may influence the achieved results. Moreover, using statistical indexes allows the use of the same evaluation parameters both in braking and traction phases. The statistical indexes are evaluated in terms of experimental $\delta v_{i}^{\text {exp }}$ and simulated $\delta v_{i}^{\text {sim }}$ absolute wheel-rail slidings defined as

$\delta v_{i}^{e x p}=\dot{x}^{e x p}-\omega_{i}^{e x p} r_{v i}, \quad \delta v_{i}^{s i m}=\dot{x}^{s i m}-\hat{\omega}_{w i} r_{v i} ;$

where $\dot{x}^{\text {exp }}$ and $\dot{\chi}^{\text {sim }}$ are the railway vehicle reference speeds, $\omega_{i}^{\exp }$ and $\hat{\omega}_{w i}$ represent the $\mathrm{i}$-th wheelset angular velocities (both in experimental and simulated cases) and $r_{v i}$ is the radius of the vehicle wheels. Absolute slidings are positive for braking phase and negative for traction one.

The statistical quantities defined in Eqs. (23) and (24) are used to make a more accurate comparison between the experimental and the simulated tests:

1. mean axle sliding $\bar{\delta} v_{i}^{\text {exp,sim }}$ : it represents the arithmetic mean of the absolute wheel-rail slidings for each wheelsets

$$
\bar{\delta} v_{i}^{\text {exp sim }}=\frac{1}{T} \int_{0}^{T} \delta v_{i}^{\text {exp }, \text { sim }} d t
$$

where $i$-th is referred to each wheelset;

2. standard deviation of axle profiles $\Delta^{\text {expsim }}$ : it defines the variability of the sliding compared with the mean value $\bar{\delta} v_{i}^{\text {exp,sim }}$

$$
\Delta^{\text {exp }, \text { sim }}=\sqrt{\frac{1}{T} \int_{0}^{T}\left(\delta_{i}^{\text {exp }, \text { sim }}-\bar{\delta} v_{i}^{\text {exp,sim }}\right)^{2} d t .}
$$

\subsection{Braking test}

The experimental data of the braking tests under degraded adhesion condition were provided by Trenitalia as a result of WSP performance evaluations. The presented results (Figs. 11 and 12) are referred to two different tests performed with the same external conditions: first of all, the adhesion coefficient $\mu_{c}$ measured on the rail (the degraded adhesion conditions are reproduced using standardized contaminant solutions of water and tensio-actives) and the vehicle characteristics such as the type of vehicle, the starting speed $V_{i n}$ and the torque $C$ applied to the wheels (Table 2). In this case, the differences between the two tests are very small and are probably due to the uncertainties and the non-linearity of the degraded adhesion phenomena.

\subsection{Traction tests}

Even the traction experimental data are coming from traction tests under degraded adhesion conditions performed by Trenitalia. The tests were carried out to analyze the performance of the AntiSkid device. The presented results are referred to two different tests (Figs. 13 and 14) evaluated with the same external and vehicle conditions. As defined for the braking tests, the external condition is the adhesion coefficient $\mu_{c}$ measured on the rail while

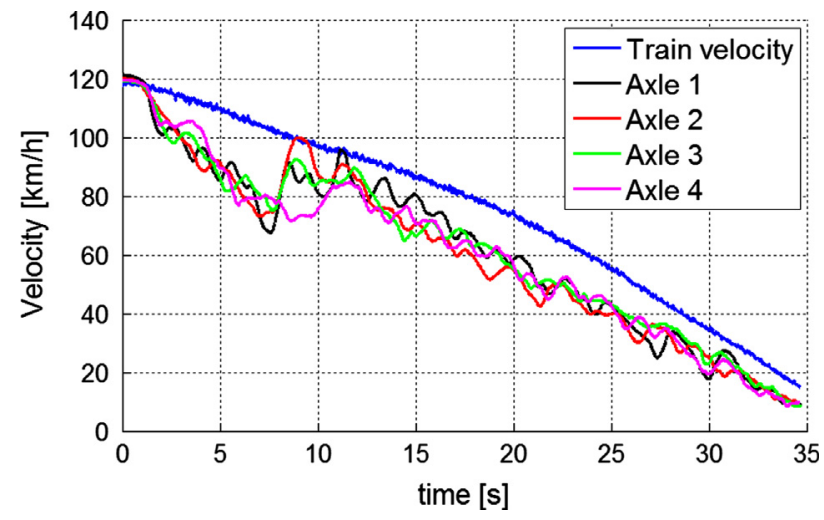

Fig. 11. Test B1: experimental train $v_{t}^{\text {exp-b1}}$ and axles velocities $v_{i}^{\text {exp-b1 }}$.

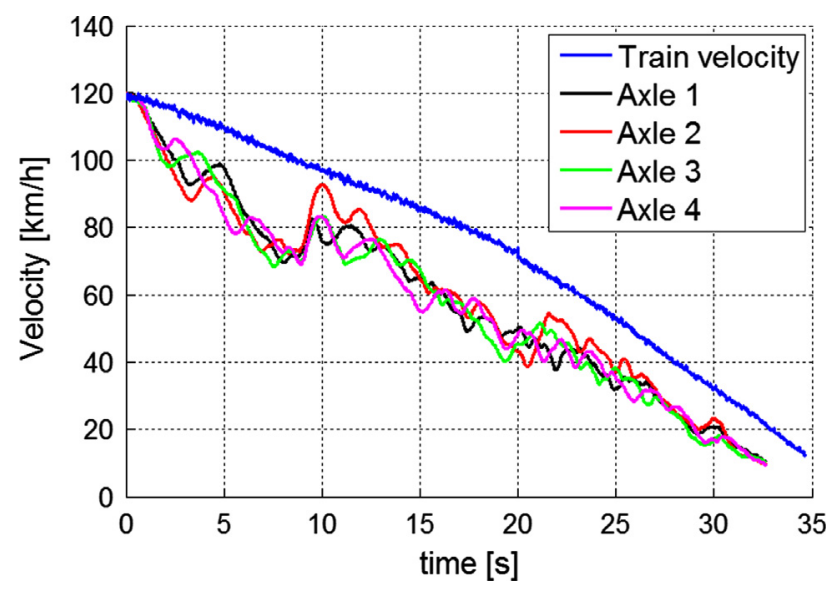

Fig. 12. Test B2: experimental train $v_{t}^{\text {exp-b1}}$ and axles velocities $v_{i}^{\text {exp-b1}}$.

Table 2

Braking test conditions.

\begin{tabular}{ll}
\hline Test parameters & Value \\
\hline Adhesion coefficient & $\mu_{c}^{s p}=0.05$ \\
Type of vehicle & UIC-Z1 \\
Wheel radius & $r_{v 1}=0.46 \mathrm{~m}$ \\
Starting speed & $V_{\text {in }}=120 \mathrm{~km} / \mathrm{h}$ \\
Braking torque & $C=9500 \mathrm{Nm}$ \\
\hline
\end{tabular}

the vehicle conditions are the type of vehicle, the starting speed $V_{\text {in }}$ and the torque $C$ applied by the motor to the wheels (see Table 3). In this case, the differences between the two experimental tests are clearer than those between the braking tests.

It is worth to note from the previous figures that the axle 4 is always in good adhesion condition (probably due to the cleaning effect of the precedent axles passing upon the rail).

\section{Numerical simulations}

In this section the numerical simulation results are presented. The numerical simulations have been performed to reproduce, using the HIL test rig simulator (in particular the innovative 2D adhesion model), the same dynamical behavior in terms of axles velocities, traveled spaces and statistical indexes of the experimental tests. Both in the braking part and in the traction one, the experimental data (Test B1-B2 and Test T1-T2) are referred to two different tests performed with the same well defined nominal conditions in which the obtained differences are probably due to 


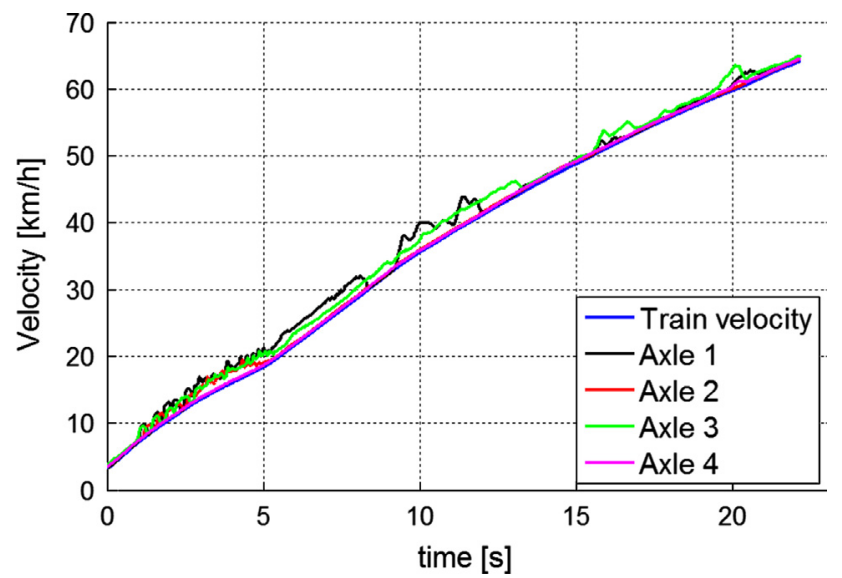

Fig. 13. Test 1 : experimental train $v_{t}^{\text {exp-t1 }}$ and axles velocity $v_{i}^{\text {exp-t1}}$.

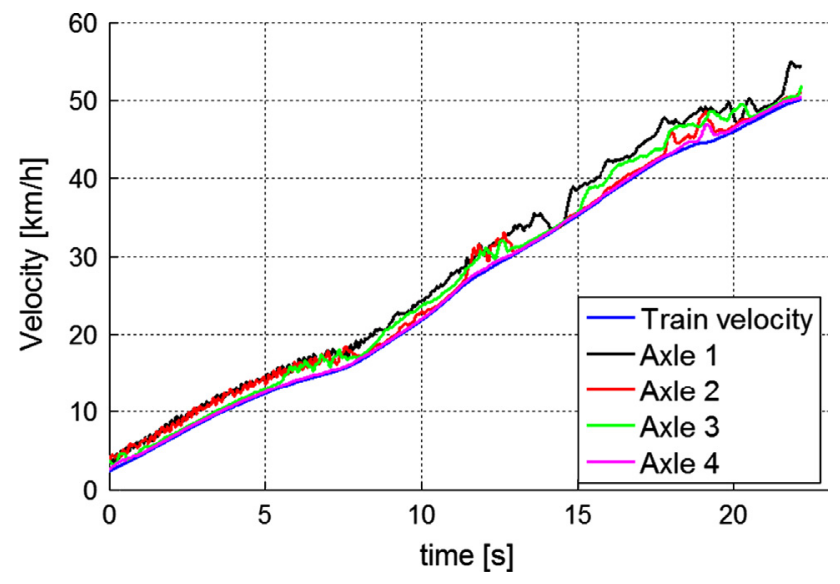

Fig. 14. Test 2: experimental train $v_{t}^{\text {exp-t2 }}$ and axles velocity $v_{i}^{\text {exp-t2 }}$.

Table 3

Traction test conditions.

\begin{tabular}{ll}
\hline Test parameters & Value \\
\hline Adhesion coefficient & $\mu_{c}^{s p}=0.06$ \\
Type of vehicle & Locomotor E402 B \\
Wheel radius $r_{v 2}$ & $0.625 \mathrm{~m}$ \\
Starting speed & $V_{i n}=5 \mathrm{~km} / \mathrm{h}$ \\
Traction torque & $C=12300 \mathrm{Nm}$ \\
\hline
\end{tabular}

the degraded adhesion phenomena non-linearity and the randomness of the environmental parameters (temperature, humidity, etc.) in the test. Moreover, in the analyzed cases, the experimental traction tests show a major sensitivity compared to the braking experimental ones.

\subsection{Numerical implementation}

The Software In the Loop (SIL) approach allows the implementation of the all HIL parts into software models. The models (hardware part, software part, controller and estimation system) are simulated in MATLAB ${ }^{\circledR}-$ Simulink $^{\circledR}$ environment. The main parameters set in the model are the integration algorithms, the type of algorithm and the step size (Table 4).

\subsection{Braking numerical simulations}

The objective of this part is to show the numerical results obtained with the HIL system simulator compared to the simulations
Table 4

Implementation parameters.

\begin{tabular}{ll}
\hline Parameter & Value \\
\hline Type of algorithm & Fixed step \\
Solver & ODE 5 (Dormand-Prince) \\
Step size & $1 e-4$ \\
\hline
\end{tabular}

Table 5

Braking simulation parameters.

\begin{tabular}{llll}
\hline & $\mu_{c}$ & $\mu_{\text {rec }}$ & $W_{\text {lim }}$ \\
\hline Value & 0.08 & 0.10 & $1.6 \mathrm{~kJ} / \mathrm{m}$ \\
\hline
\end{tabular}

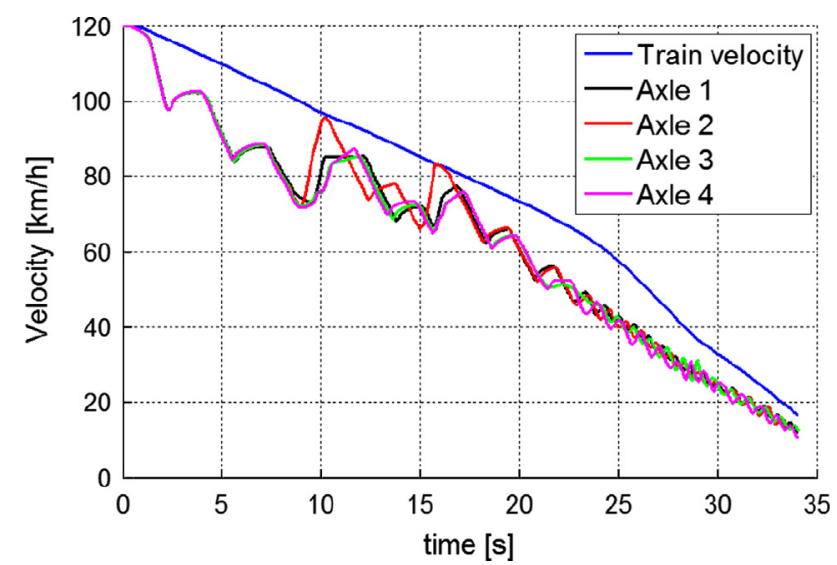

Fig. 15. Intermediate braking test: simulated train velocity $v_{t}^{\text {sim }}$ and axles velocities $v_{i}^{\text {sim }}$

of the Test B1-B2 experimental data; to obtain these results the 2D adhesion model had been tuned by means of these parameters (Table 5).

The paragraph is divided into two parts: the HIL system performances and the controller performances. The HIL system performances are related to the capacity of the system to reproduce the same velocity behavior of the experimental data. The comparison between the simulated and the experimental Test B1 results is performed by means of statistical indexes defined in the previous Section 4 and also in a qualitative way. On the contrary, the controller performances are related to aptitude of the controller to simulate the same dynamical behavior of the virtual railway vehicle model on the test rig model (expressed only in a qualitative way).

\subsubsection{HIL system performances}

As can be seen, comparing the experimental (Figs. (11) and (12)) and the simulated (Fig. 15) braking test, the differences among the axle velocities and the statistical indexes are quite small (Table 6). In Fig. 15, it is worth to note the adhesion recovery effect obtained through the adhesion model introduced in the virtual railway vehicle model; this effect is clearly visible also in the experimental data. Moreover, analyzing Figs. 16 and 17, even the results in terms of differences between experimental $v_{t}^{\text {exp-b1, }}$, $v_{t}^{\text {exp- } b 2}$ and simulated $v_{t}^{\text {sim- } b 1}$ train velocity and differences between simulated $s^{\text {sim- } b 1}, s^{\text {sim- } b 2}$ and experimental $s^{\text {exp-b1 }}$ traveled distance (Table 7) are very interesting since they confirm that the HIL test rig simulator is able to reproduce the experimental data. 
Table 6

Comparison among simulated and experimental test statistical indexes.

\begin{tabular}{lllllll}
\hline Axle & $\begin{array}{l}\bar{\delta} v_{i}^{\text {exp-b1 }} \\
(\mathrm{km} / \mathrm{h})\end{array}$ & $\begin{array}{l}\bar{\delta} v_{i}^{\text {sim-b }} \\
(\mathrm{km} / \mathrm{h})\end{array}$ & $\begin{array}{l}\bar{\delta} v_{i}^{\text {exp-b2 }} \\
(\mathrm{km} / \mathrm{h})\end{array}$ & $\begin{array}{l}\Delta^{\text {exp }-b 1} \\
(\mathrm{~km} / \mathrm{h})\end{array}$ & $\begin{array}{l}\Delta^{\text {sim-b }} \\
(\mathrm{km} / \mathrm{h})\end{array}$ & $\begin{array}{l}\Delta^{\text {exp-b2 }} \\
(\mathrm{km} / \mathrm{h})\end{array}$ \\
\hline Axle 1 & 13.08 & 12.82 & 12.78 & 6.45 & 6.56 & 6.58 \\
Axle 2 & 13.36 & 12.38 & 12.06 & 7.25 & 6.95 & 6.86 \\
Axle 3 & 13.09 & 13.32 & 13.20 & 5.92 & 5.70 & 5.63 \\
Axle 4 & 13.52 & 13.59 & 13.43 & 6.22 & 5.82 & 5.75 \\
\hline
\end{tabular}

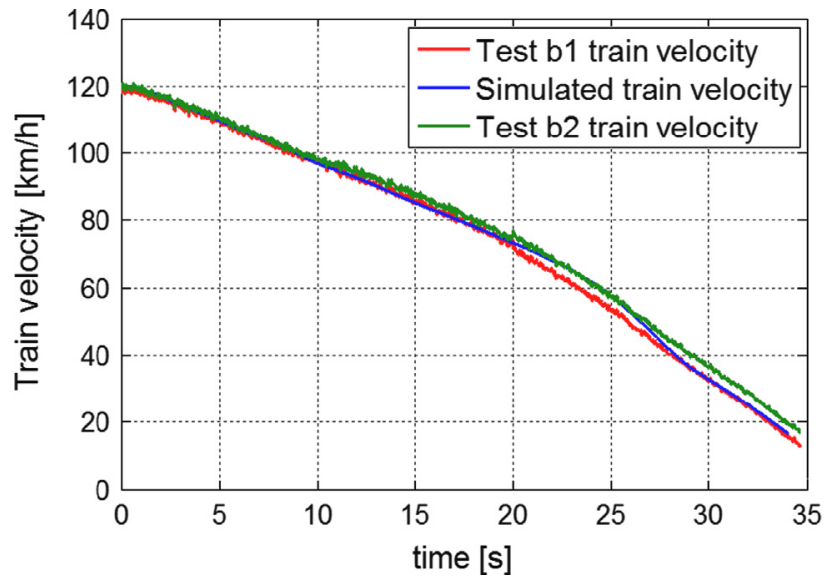

Fig. 16. Intermediate braking test: comparison among the simulated train velocity $v_{t}^{\text {sim-b }}$ and the experimental train velocities $v_{t}^{\text {exp- } b 1}-v_{t}^{\text {exp }-b 2}$.

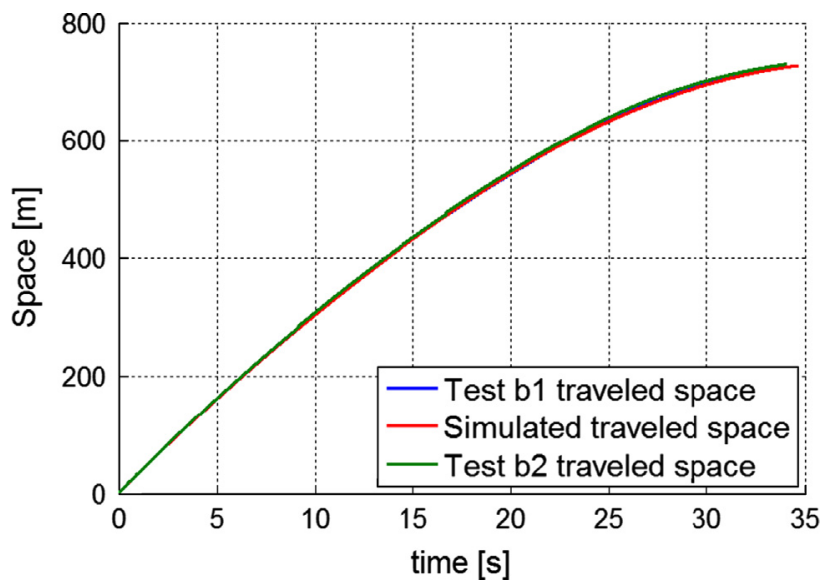

Fig. 17. Intermediate braking test: comparison among the simulated traveled distance $s^{s i m-b}$ and the experimental ones $s^{\exp -b 1}-s^{\text {exp-b2}}$.

\subsubsection{Controller performances}

Figs. 18 and 19 are related to the controller performances and, in particular, to the system capacity to reproduce the virtual railway vehicle behavior. Both the speed error $e_{\omega}$ and the torque estimation error $e_{c}$ are low, confirming the robustness of the HIL system. The controller is able to manage the initial transient (which can be also quite heavy) and to reject the disturbances due to the recovery adhesion effect (clearly visible in the second part of the figure). In Fig. 20 the estimated torque trends $\hat{C}_{s}$ are shown (the torques are negative since they are in braking phase) and it is possible to see both the WSP interventions and the increase of the torque due to the recovery adhesion effect at the end of the simulation. Whereas, in Figs. 21 and 22, the creep forces and the comparison among the simulated and the measured adhesion coefficients are displayed (referred to the second wheelset). In the last part of the braking phase, it is very interesting to

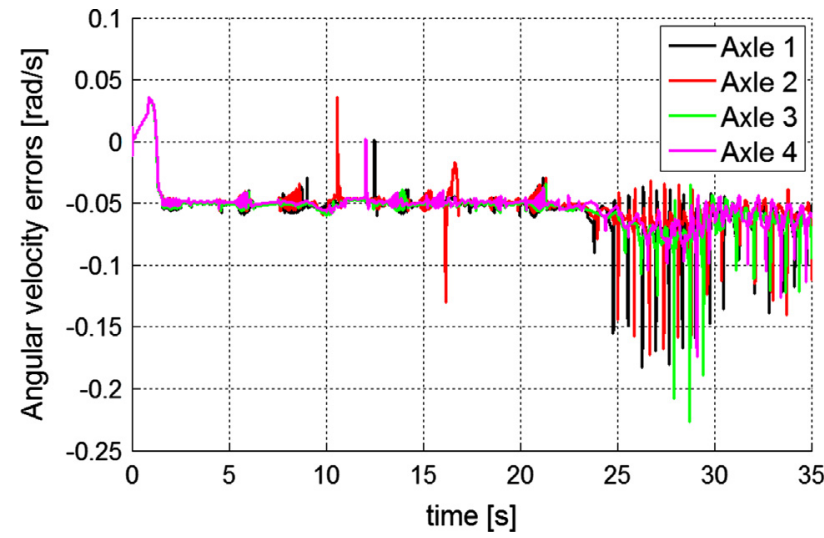

Fig. 18. Intermediate braking test: speed errors $e_{\omega}$ between the simulated wheelset angular velocity $\omega_{w s}$ and the measured wheelset angular velocity $\hat{\omega}_{w}$.

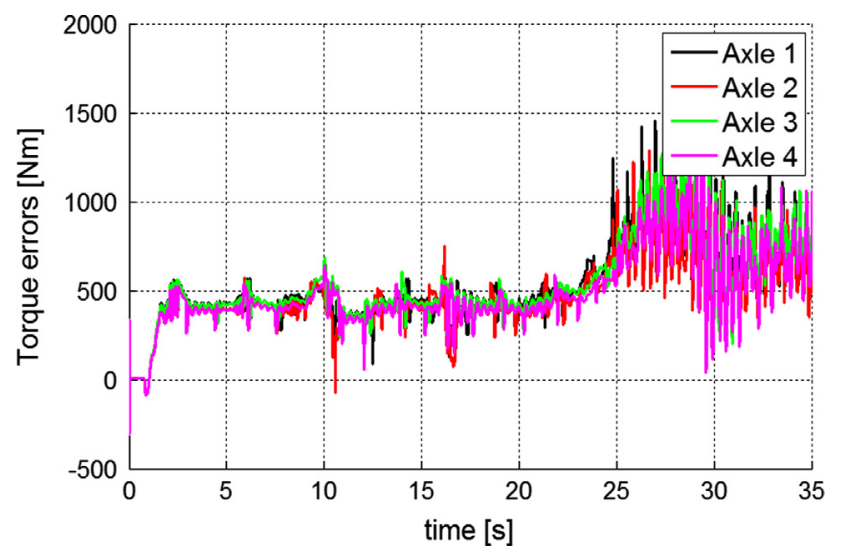

Fig. 19. Intermediate braking test: torque estimation error $e_{c}$ between the simulated torque value $C_{s}$ and the measured one $\hat{C}_{s}$.

observe the behavior of the adhesion coefficient, where it is distinctly visible the increasing of the coefficient due to the recovery adhesion effect.

\subsection{Traction numerical simulations}

The aim of this part is to show the numerical results obtained with the HIL system simulator in terms of accuracy but, in this case, as regards the traction phase. The tuning of the $2 \mathrm{D}$ adhesion model allows the achievement of good results in terms of evaluation of the interesting physical parameters (Table 8).

The paragraph is divided into two parts: the HIL system performances and the controller performances. In particular, in the simulated test, the authors obtained a numerical simulation with intermediate characteristics between Test T1 and Test T2 (in terms of velocities and statistical indexes). According to the proposed HIL architecture, the controller performances are related to the controller capacity to reproduce the same dynamical behavior of the virtual railway vehicle model on the test rig model.

\subsubsection{HIL system performances}

Fig. 23 and Table 9 have to be compared with experimental data (Figs. 13 and 14) to analyze the differences in terms of mean axle sliding and standard deviation of axle profiles. As it can be observed, the differences in terms of trends and statistical indexes are quite small. From a general point of view, the innovative 2D adhesion model allows an accurate reproduction of the experimental behavior in terms of slidings and perceived adhesion coefficient; in particular, according to the traction experimental 


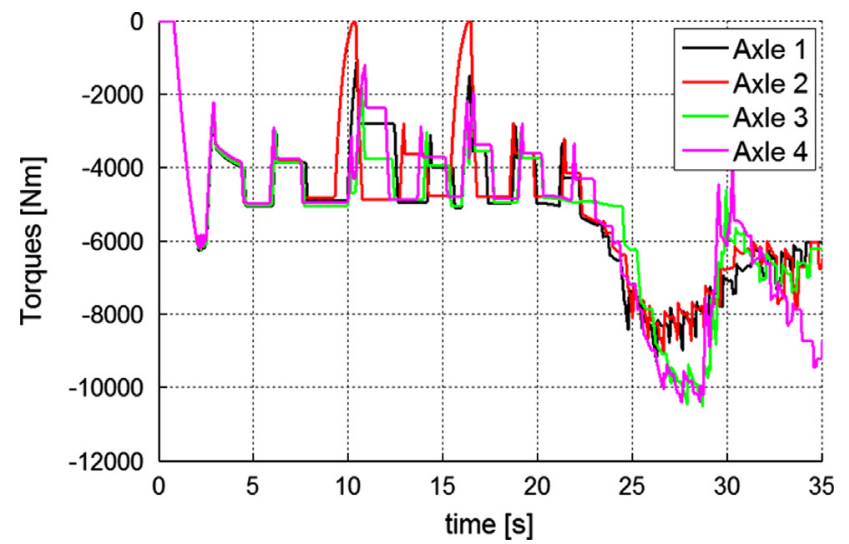

Fig. 20. Intermediate braking test: estimated torques $\hat{C}_{s}$ evaluated by the estimator blocks.

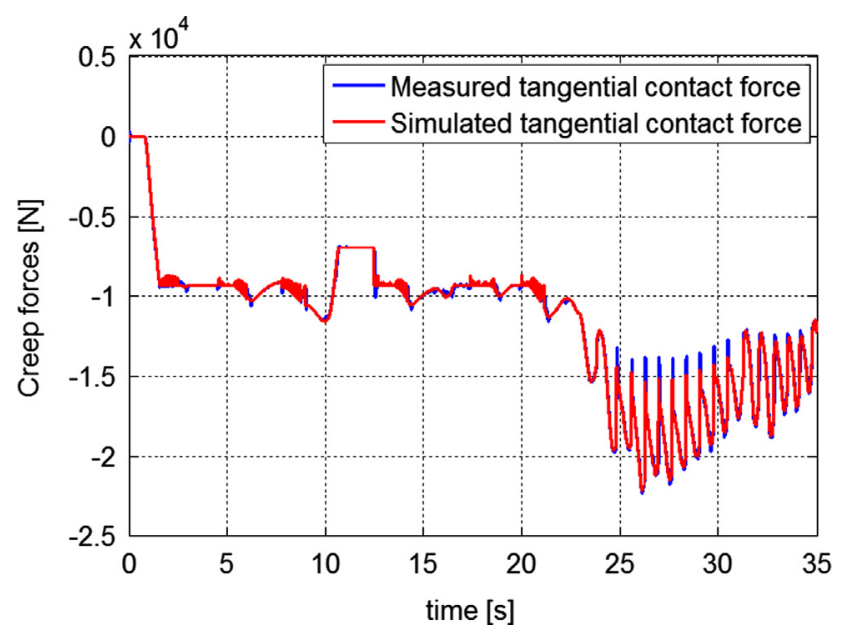

Fig. 21. Intermediate braking test: behavior of measured creep forces $T_{\text {mis }}$ and simulated ones $T_{\text {sim }}$

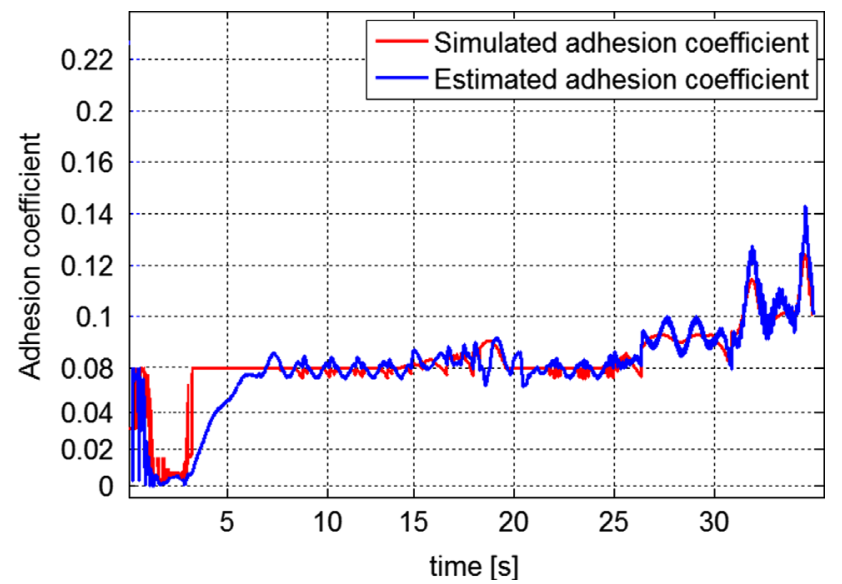

Fig. 22. Intermediate braking test: comparison among the simulated $\mu_{\text {sim }}$ and the measured $\mu_{\text {mis }}$ adhesion coefficients.

data, the slidings of the fourth wheelset are quite small (because of the better adhesion conditions and the load distribution). In the analyzed cases, it is possible to observe that the slidings in the traction phase are less than the braking phase slidings.

Even Figs. 24 and 25 and Table 10 prove the efficiency of the system to reproduce an intermediate dynamical behavior among the Test T1 and the Test T2 in terms of velocity trends and statistical indexes.
Table 7

Traveled distances.

\begin{tabular}{ll}
\hline Test & Traveled distance $(\mathrm{m})$ \\
\hline Experimental Test B1 $s^{\text {exp-b1 }}$ & 727.53 \\
Simulated Test B1 $s^{\text {sim-b }}$ & 724.88 \\
Experimental Test B2 $s^{\text {exp-b2 }}$ & 722.65 \\
\hline
\end{tabular}

Table 8

Traction simulation parameters.

\begin{tabular}{lccc}
\hline & $\mu_{c}$ & $\mu_{\text {rec }}$ & $W_{\text {lim }}$ \\
\hline Value & 0.09 & 0.10 & $1.6 \mathrm{~kJ} / \mathrm{m}$ \\
\hline
\end{tabular}

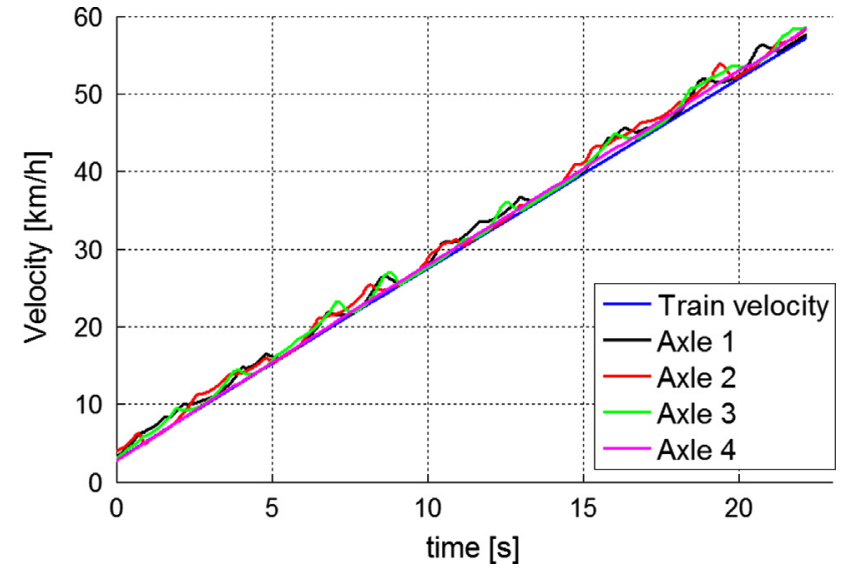

Fig. 23. Intermediate traction test: simulated train velocity $v_{t}^{\text {sim-t }}$ and axle velocities $v_{i}^{\text {sim- } t}$.

Table 9

Traction simulation parameters.

\begin{tabular}{lllllll}
\hline Axle & $\bar{\delta} v_{i}^{\text {exp-t1 }}$ & $\begin{array}{l}\bar{\delta} v_{i}^{\text {sim-t }} \\
(\mathrm{km} / \mathrm{h})\end{array}$ & $\begin{array}{l}\bar{\delta} v_{i}^{\text {exp-t2 }} \\
(\mathrm{km} / \mathrm{h})\end{array}$ & $\begin{array}{l}\Delta^{\text {exp- } 1} \\
(\mathrm{~km} / \mathrm{h})\end{array}$ & $\begin{array}{l}\Delta^{\text {sim-t }} \\
(\mathrm{km} / \mathrm{h})\end{array}$ & $\begin{array}{l}\Delta^{\text {exp }-\mathrm{t} 2} \\
(\mathrm{~km} / \mathrm{h})\end{array}$ \\
\hline Axle 1 & -1.44 & -1.56 & -2.59 & 1.21 & 0.73 & 0.96 \\
Axle 2 & -0.99 & -1.07 & -1.23 & 0.48 & 0.74 & 0.86 \\
Axle 3 & -1.49 & -1.38 & -1.37 & 0.69 & 0.77 & 0.88 \\
Axle 4 & -0.34 & -0.48 & -0.29 & 0.10 & 0.33 & 0.24 \\
\hline
\end{tabular}

\subsubsection{Controller performances}

Figs. 26 and 27 represent the controller performances: both the speed error $e_{\omega}$ and the torque estimation error $e_{c}$ are quite small; therefore, even in the traction phase, the controller is very robust and allows the stabilization of the initial transient. The estimated torques showed in Fig. 28 underline the antiskid behavior, with the 4th axle in full adhesion condition (according to the experimental data behavior) caused by the load transfer during traction phase and the cleaning effect of the rail surface by means of the passing of the previous axles (see Figs. 13, 14-23). In Figs. 29 and 30 the creep forces and the comparison between the simulated and the measured adhesion coefficients are displayed (referred to the second wheelset, such as in the braking phase); in this case the behavior of the adhesion coefficient shows that the recovery adhesion effect is not reached, probably, due to the low energy reached caused by the lower slidings if compared to the braking phases (see Section 3.2). 


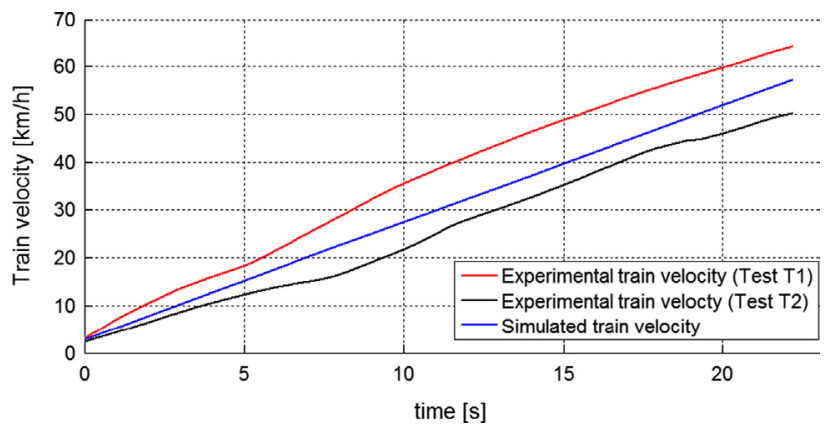

Fig. 24. Comparison among the simulated train velocity $v_{t}^{\text {sim- } t}$ and the experimental train velocities $v_{t}^{\text {exp-t1 }} v_{t}^{\text {exp }-t 2}$.

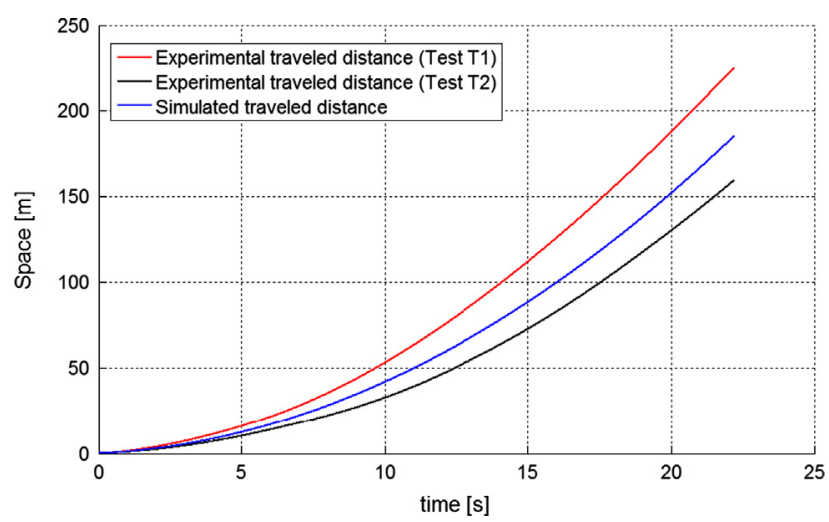

Fig. 25. Comparison among the simulated traveled distance $s^{\text {sim-t }}$ and the experimental ones $s^{\text {exp- } t 1} s^{\text {exp-t2}}$.

Table 10

Traveled distances.

\begin{tabular}{ll}
\hline Test & Traveled distance $(\mathrm{m})$ \\
\hline Test $1 s^{\text {exp- } t 1}$ & 225.57 \\
Simulated Test $s^{\text {sim- } t}$ & 185.37 \\
Test $2 s^{\text {exp- } t 2}$ & 159.64 \\
\hline
\end{tabular}

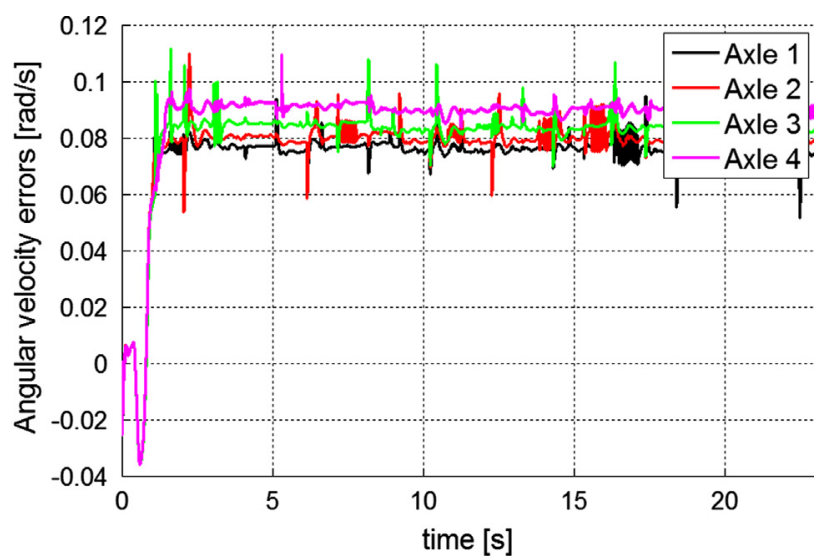

Fig. 26. Speed errors $e_{\omega}$ between the wheelset simulated angular velocity $\omega_{w s}$ and the wheelset measured angular velocity $\hat{\omega}_{w}$.

\section{Conclusions and further developments}

In this paper the authors presented an innovative complete model of a HIL railway vehicle test rig (using Software In the Loop approach) which allows the simulation of degraded adhesion

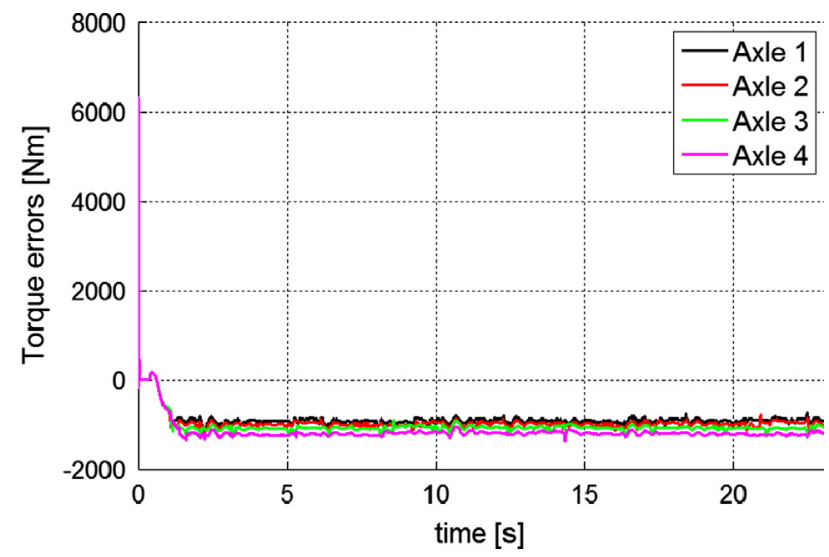

Fig. 27. Torque estimation errors $e_{c}$ between the simulated torque value $C_{s}$ and the estimated one $\hat{C}_{s}$.

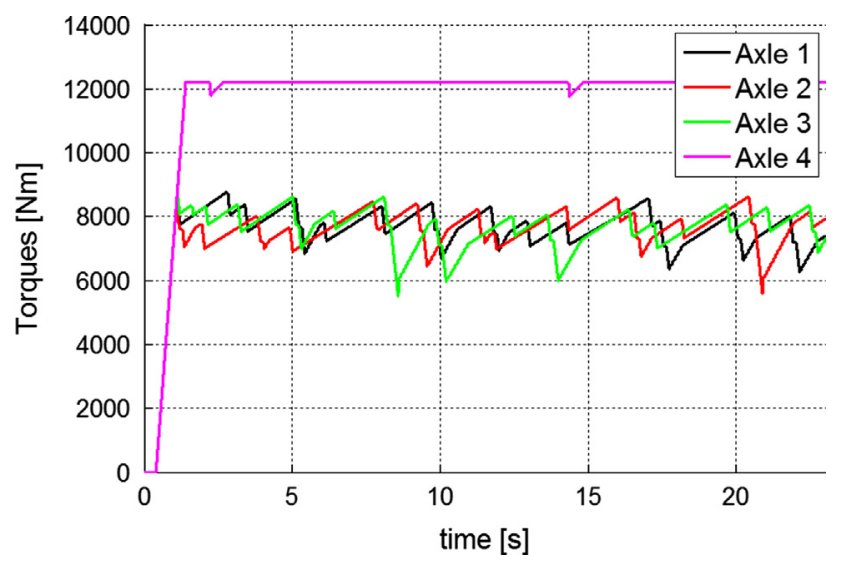

Fig. 28. Estimated torques $\hat{C}_{s}$ evaluated by the estimator blocks.

conditions. The SIL approach is very useful because it permits to preliminary analyze the behavior of the system before its physical construction; moreover it allows the reduction of the economic investments since all the components of the system are simulated. The HIL system includes the hardware part (railway vehicle and roller rig), the software part (the virtual railway vehicle model), the controller part (the controller) and the estimator part (the torque estimator), according to the architecture presented in Fig. 2. The HIL roller rig system is inspired by the Osmannoro test rig recently built by Trenitalia in Florence and actually under pre-test activities.

The HIL architecture has been developed to simulate degraded adhesion conditions on the roller rig, using a previous innovative approach proposed in [3] but simulated only through 2D simplified model of the test rig and without experimental validation. In this work, the authors, have considered experimental data acquired by Trenitalia S.p.a. during safety on board subsystem on-track tests both in braking and traction phases. These tests are evaluated on two different railway vehicles as regards the braking and the traction phases, respectively when degraded adhesion conditions occur. The most important results obtained in this research activity, compared to previous works [3-6], are the implementation in the HIL system of the complete 3D multibody models of the roller rig and of the vehicles (inspired on the real characteristics) and of innovative contact models (both the 3D contact model for revolute surfaces and 2D adhesion model in the virtual railway vehicle model); the 2D adhesion model is based on energetic criteria which allow the recovery adhesion effect; in addition, in the vehicle model, both traction and braking on board devices (respectively antiskid and WSP) with nearly realistic 


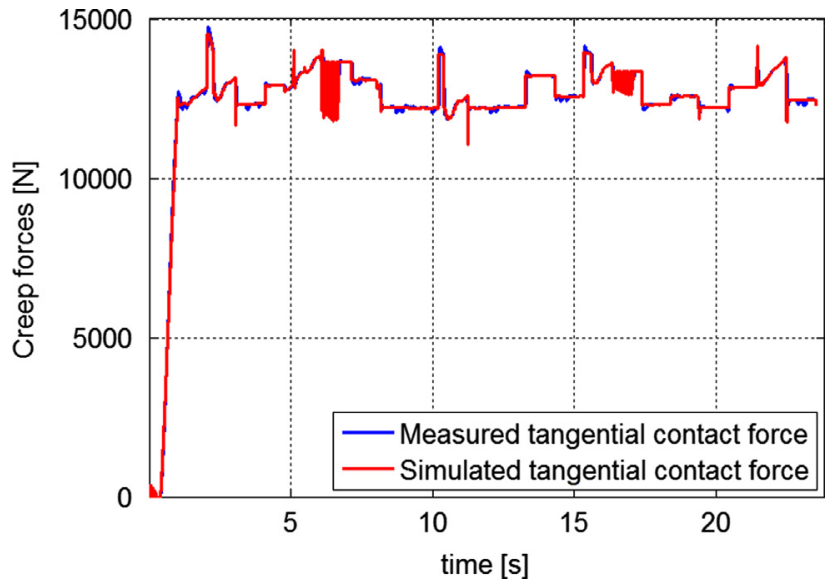

Fig. 29. Intermediate traction test: behavior of measured creep forces $T_{m i s}$ and simulated ones $T_{\text {sim }}$.

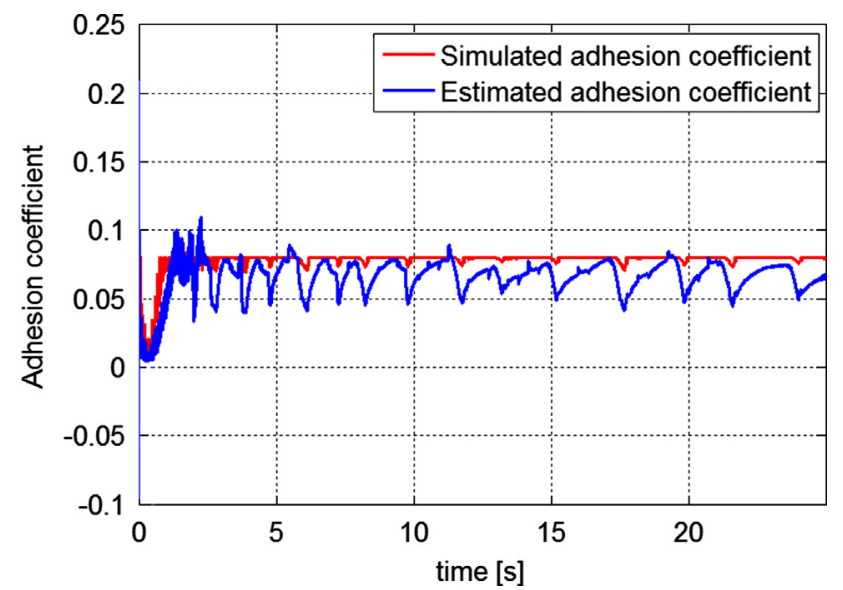

Fig. 30. Intermediate traction test: comparison among the simulated $\mu_{\text {sim }}$ and the measured $\mu_{\text {mis }}$ adhesion coefficients.

behavior are modeled. Finally, the extension of the controller law with a sliding mode approach which permitted the achievement of the stability and the robustness of the whole HIL system.

In the numerical simulation chapter, the HIL performance and the controller performance are analyzed; the results are quite cheering. The statistical indexes and the simulated behavior are really similar to the experimental data thanks to the adhesion model and the HIL architecture; moreover, even the robustness of the controller during the initial transient and the recovery adhesion phase is showed. The further developments of this work are related to the pre-testing activities of the real Osmannoro HIL system and will be the comparison between the results of this HIL system model with the experimental data of the real HIL Osmannoro test rig. The next intermediate step of the research activity is to obtain in the MATLAB ${ }^{\circledR}$-Simulink ${ }^{\circledR}$ environment a validated numerical model of the Osmannoro HIL system which can carry out preliminary tests and the analyses of difference control strategies. The final purpose is the implementation of the controller and the virtual railway vehicle model directly within the Osmannoro rollerrig hardwares.

\section{References}

[1] S. Iwnicki, P. Allen, Handbook of Railway Vehicle Dynamics, CRC/Taylor and Francis, Boca Raton, FL, 2006.

[2] A. Jaschinki, H. Chollet, S. Iwnicki, The application of the roller rigs to railway vehicle dynamics, Vehicle System Dynamics 31 (1999) 345-392.

[3] M. Malvezzi, B. Allotta, L. Pugi, Feasibility of degraded adhesion tests in a locomotive roller rig, Proceedings of the Institution of Mechanical Engineering Part F (2008) 22-27.

[4] R. Dukkipati, A parametric study of the lateral stability of a railway bogie on a roller rig, Proceedings of the Institution of Mechanical Engineering Part F 213 (1999) 39-47.

[5] L. Pugi, M. Malvezzi, A. Tarasconi, A. Palazzolo, G. Cocci, M. Violani, HIL simulation of WSP systems on MI-6 test rig, Vehicle System Dynamics 44 (2006) 843-852.

[6] R. Conti, E. Meli, L. Pugi, M. Malvezzi, F. Bartolini, B. Allotta, A. Rindi, P. Toni, A numerical model of a HIL scaled roller rig for simulation of wheel-rail degraded adhesion condition, Vehicle System Dynamics 50 (2012) 775-804.

[7] B. Allotta, L. Pugi, F. Bartolini, F. Cangioli, A scaled roller test rig for high speed vehicles, Vehicle System Dynamics 48 (2010) 3-18.

[8] B. Allotta, R. Conti, M. Malvezzi, E. Meli, L. Pugi, A. Ridolfi, Numerical simulation of a HIL full scale roller-rig model to reproduce degraded adhesion conditions in railway applications, Proceedings of ECCOMAS 2012, 2012, PP. 258-263.

[9] B. Allotta, L. Pugi, A. Ridolfi, M. Malvezzi, G. Vettori, A. Rindi, Evaluation of odometry algorithm performance using railway vehicle dynamic model, Vehicle System Dynamics 50 (2012) 699-724.

[10] F. U. 541-05, Brakes-Specification for the Construction of Various Brake PartsWSP, 2005.

[11] Technical Description of E402B Antiskid System, ADtranz, 1997.

[12] L. Pugi, Progettazione e realizzazione di un banco prova per lo studio e l'omologazione di sistemi di odometria ferroviari, volume, Ph.D. Dissertation, University of Bologna, 2003.

[13] M. Malvezzi, E. Meli, J. Auciello, S. Falomi, Dynamic simulation of railway vehicles: wheel-rail contact analysis, Vehicle System Dynamics 47 (2009) 867-899.

[14] M. Malvezzi, E. Meli, A. Rindi, S. Falomi, Determination of wheel-rail contact points with semianalytic method, Multibody System Dynamics 20 (2008) 327-358.

[15] J. Slotine, W. Li, Applied Nonlinear Control, Prentice Hall, 1991.

[16] B. Allotta, M. Malvezzi, E. Meli, L. Pugi, A. Ridolfi, A. Rindi, G. Vettori, Simulation of railway braking tests under degraded adhesion conditions, Proceedings of the IMSD2012, 2012.

[17] J. Kalker, Three-dimensional Elastic Bodies in Rolling Contact, Kluwer Academic Publishers, Dordrecht, Netherlands, 1990.

[18] O. Polach, Creep forces in simulations of traction vehicles running on adhesion limit, Wear 258 (2005) 992-1000.

[19] K. Johnson, Contact Mechanics, Cambridge University Press, Cambridge, England, 1985.

[20] S. Falomi, M. Malvezzi, E. Meli, Multibody modeling of railway vehicles: innovative algorithms for the detection of wheel-rail contact points, Wear 271-1 (2011) 453-461.

[21] S. Magheri, M. Malvezzi, E. Meli, A. Rindi, An innovative wheel-rail contact model for multibody applications, Wear 271-1 (2011) 462-471.

[22] U.-E. 15595, Railway Applications, Braking-Wheel Sliding Protection, 2009.

[23] U.B.C. Group, Braking Questions Methodology for the Safety Margin ETCS/ ERTMS dt414, 2006.

[24] P. Blau, Embedding wear models into friction models, Tribology Letters 34 (2009) 75-79. 